\title{
Anti-inflammatory and Anti-ulcer Activities of New Fused Thiazole Derivatives Derived from 2-(2-Oxo-2H-chromen-3-yl)thiazol-4(5H)-one
}

\author{
Rafat M. Mohareb, ${ }^{1 *}$ Fatima Al-Omran, ${ }^{2}$ Mahmoud A. Abdelaziz ${ }^{3}$ \\ and Rehab A. Ibrahim ${ }^{4}$ \\ ${ }^{1}$ Department of Chemistry, Faculty of Science, Cairo University, Giza, A. R. Egypt \\ ${ }^{2}$ Department of Chemistry, Faculty of Science, Kuwait University, P. O. Box 12613, Safat 13060, Kuwait \\ ${ }^{3}$ Chemistry Department, Faculty of Science, Tabuk university, Tabuk 71491, P. O. Box 741, Kingdom of Saudi Arabia \\ ${ }^{4}$ Higher Institute of Engineering and Technology, El-Tagammoe El-Khames, New Cairo, A. R. Egypt \\ *Corresponding author: E-mail: raafat_mohareb@yahoo.com
}

Received: 12-01-2017

\begin{abstract}
The reaction of the 2-(4-oxo-4,5-dihydrothiazol-2-yl)acetonitrile (1) with salicylaldehyde (2) in 1,4-dioxane containing a catalytic amount of piperidine gave the coumarin derivative $\mathbf{3}$. The latter reacted with different reagents to give pyrano[4,5- $b]$ thiazole, pyrido[4,5-b]thiazole and thieno[5,4-b]thiazole dereivatives. The anti-inflammatory and anti-ulcer activities of the newly synthesized products were evaluated and the results showed that compounds $7 \mathbf{a}, \mathbf{8 a}, \mathbf{1 0 b}, \mathbf{1 3 b}$, 15b, 18a, 19b, 19c, and 19d showed higher activity compared to the rest of the compounds. In addition to this, toxicity of such active compounds was studied against shrimp larvae where compounds 10b, 18a, 19c and 19d showed to be non-toxic against the tested organisms.
\end{abstract}

Keywords: 4,5-dihydrothiazol, coumarin, pyrimidine, anti-inflammatory, anti-ulcer activity

\section{Introduction}

Thiazole is a core structural motif present in a variety of natural products, such as vitamin B1 (thiamine) and penicillin. Thiazole derivatives also exhibit a broad spectrum of medicinal and biological properties, such as antibacterial, antifungal, ${ }^{1}$ anti-inflammatory, ${ }^{2}$ antiviral, ${ }^{3}$ antimalarial, ${ }^{4}$ and anti-HIV activities. ${ }^{5}$ Thiazole analogs have also been reported as ligands at estrogen receptors, ${ }^{6}$ neuropeptides, ${ }^{7}$ Y5 adenosine receptors, ${ }^{8}$ and act as inhibitors of human platelet aggregation factor, ${ }^{9}$ urokinase, ${ }^{10}$ and poly(ADP-ribose) polymerase- $1 .{ }^{11}$ Selenazoles have been reported to possess antibacterial properties, ${ }^{12}$ superoxide-anion-scavenging activity, ${ }^{13}$ and exhibit cytotoxicity and DNA fragmentation effects in human HT-1080 fibrosarcoma cells. ${ }^{14}$ The structures of sulfathiazole, meloxicam, and selenazofurin and their pharmacological activities are given in Fig. 1.<smiles>Nc1ccc(S(=O)(=O)Nc2nccs2)cc1</smiles>

Sulfathiazole (antibacterial)<smiles>Cc1cnc(NC(=O)C2=C(O)c3ccccc3S(=O)(=O)N2C)s1</smiles>

Meloxican (antiinflammatory<smiles>NC(=O)c1c[se]c(C2OC(CO)C(O)C2O)n1</smiles>

Selenazofurin (antibacterial)

Fig 1. Biologically active thiazole and senazole drivatives 
In many report structural modification of the heterocyclic rings through the construction of new heterocyclic nuclei enhances the pharmaceutical applications of the resulting molecules. ${ }^{15,16}$ This encouraged our efforts in this work to modify 2-(4-oxo-4,5-dihydrothiazol-2yl)acetonitrile through its reaction with salicyladehyde to produce a chromen-3-yl)thiazol which was used as the key starting compound for many further heterocyclic transformations. The anti-inflammatory and anti-ulcer evaluations of the newly synthesized compounds were studied.

\section{Results and Discussion}

In the present work the 2-(4-oxo-4,5-dihydrothiazol-2-yl)acetonitrile (1) reacted with salicylaldehyde (2) in 1,4-dioxane containing a catalytic amount of piperidine to give the coumarin derivative $\mathbf{3}$. The structure of $\mathbf{3}$ was based on its analytical and spectral data. Thus, the ${ }^{1} \mathrm{H}$ NMR spectrum showed a singlet at $\delta 4.28 \mathrm{ppm}$ equivalent to the thiazole $\mathrm{CH}_{2}$ group, a singlet at $\delta 6.52 \mathrm{ppm}$ indicating the coumarin $4 H$, a multiplet at $\delta 7.28-7.38 \mathrm{ppm}$ for the $\mathrm{C}_{6} \mathrm{H}_{4}$ group. Moreover, the ${ }^{13} \mathrm{C}$ NMR spectrum sho-<smiles>N#CCC1=NC(=O)CS1</smiles>

1<smiles>O=Cc1ccccc1O</smiles>

2

\section{$\underset{\text { piperidine }}{\stackrel{\text { 1,4-dioxane }}{\longrightarrow}}$}

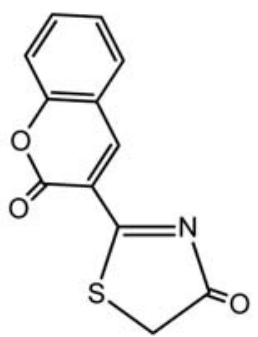

3<smiles>[X]c1ccc(C2C(C#N)=C(N)Oc3nc(-c4cc5ccccc5oc4=O)sc32)cc1</smiles>

6a, $\mathrm{X}=\mathrm{H}$

b, $\mathrm{X}=\mathrm{Cl}$

c, $\mathrm{X}=\mathrm{OCH}_{3}$<smiles>O=C1CSC(c2cc3ccccc3oc2=O)=N1</smiles>

3<smiles>N#CCC#N</smiles>

$$
\begin{aligned}
\text { 5a, } X & =\mathrm{H} \\
\mathbf{b}, \mathrm{X} & =\mathrm{Cl} \\
\mathbf{c}, \mathrm{X} & =\mathrm{OCH}_{3}
\end{aligned}
$$

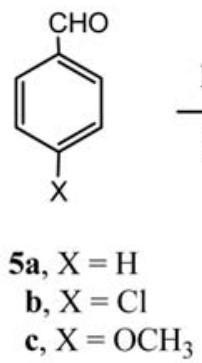

$\mathrm{EtOH}$

$\mathrm{NH}_{4} \mathrm{OAc}$<smiles>[X]c1ccc(C2C(C#N)=C(N)Nc3nc(-c4cc5ccccc5oc4=O)sc32)cc1</smiles>

7a, $\mathrm{X}=\mathrm{H}$

b, $\mathrm{X}=\mathrm{Cl}$

c, $\mathrm{X}=\mathrm{OCH}_{3}$ 
wed beside the expected signals, $\delta 58.6 \mathrm{ppm}$ indicating the presence of the thiazole $\mathrm{CH}_{2}$, two signals at $\delta 164.3$, $168.6 \mathrm{ppm}$ equivalent to the two $\mathrm{CO}$ groups and a signal at $\delta 170.3 \mathrm{ppm}$ for the $\mathrm{C}=\mathrm{N}$.

Compound $\mathbf{3}$ underwent multi-component reactions through its transformation with malononitrile (4) and any of benzaldehyde (5a), 4-chlorobenzaldehyde (5b) or 4methoxybenzaldehyde (5c) to give the 5-amino-7 $\mathrm{H}$-pyrano[2,3- $d]$ thiazole-6-carbonitrile derivatives $\mathbf{6 a - c}$, respectively. On the other hand, carrying out the same reaction, but using ammonium acetate instead of piperidine gave the pyrido[2,3- $d]$ thiazole derivatives $7 \mathbf{a}-\mathbf{c}$, respectively (Scheme 1).

Compound $\mathbf{3}$ was ready for thiophene formation through the well known Gewald's thiophene synthesis. ${ }^{17,18}$ Thus, the reaction of $\mathbf{3}$ with elemental sulfur and either malononitrile (4) or ethyl cyanoacetate (4') gave the thieno[2,3- $d]$ thiazole derivatives $\mathbf{8 a}$ and $\mathbf{8 b}$, respectively. The analytical and spectral data of $\mathbf{8 a}, \mathbf{b}$ were the tools of their structural elucidation. Thus, the ${ }^{1} \mathrm{H}$ NMR spectrum of $\mathbf{8 a}$ showed a signal at $\delta 4.93$ ppm equivalent to the $\mathrm{NH}_{2}$ group ( $\mathrm{D}_{2} \mathrm{O}$ exchangeable), a singlet at $\delta 6.62 \mathrm{ppm}$ indica-<smiles>O=C1CSC(c2cc3ccccc3oc2=O)=N1</smiles>

3<smiles>[X]c1c(N)sc2sc(-c3cc4ccccc4oc3=O)nc12</smiles>

$\mathbf{8 a}, \mathrm{X}=\mathrm{CN}$

b, $\mathrm{X}=\mathrm{COOEt}$

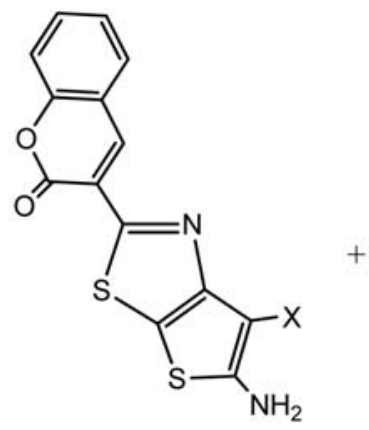

$\mathbf{8 a}, \mathrm{X}=\mathrm{CN}$

b, $\mathrm{X}=\mathrm{COOEt}$

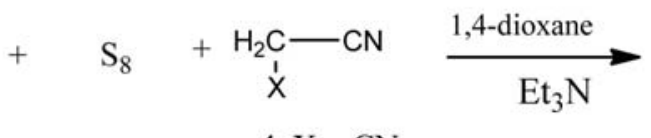

4, $\mathrm{X}=\mathrm{CN}$

$4^{\prime}, \mathrm{X}=\mathrm{COOEt}$

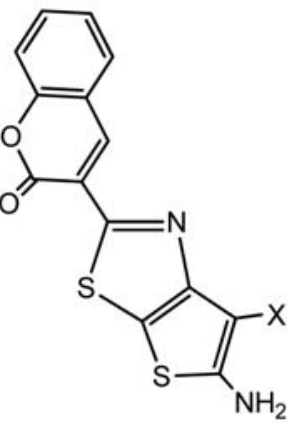

8a, $\mathrm{X}=\mathrm{CN}$

b, $\mathrm{X}=\mathrm{COOEt}$
PhNCS

9

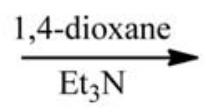

$=$

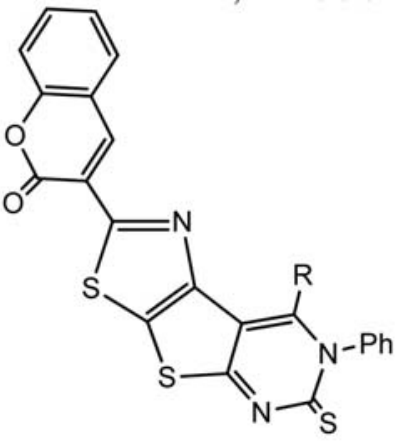

10a, $\mathrm{R}=\mathrm{NH}_{2}$

b, $\mathrm{R}=\mathrm{OH}$

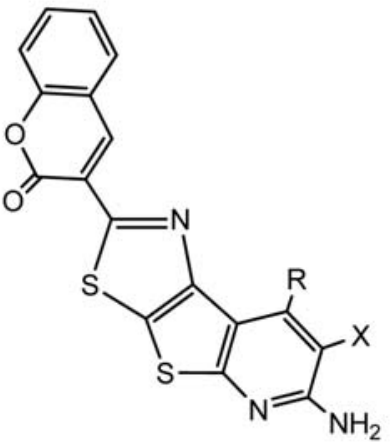

11a, $\mathrm{R}=\mathrm{NH}_{2}, \mathrm{X}=\mathrm{CN}$

b, $\mathrm{R}=\mathrm{OH}, \mathrm{X}=\mathrm{CN}$

c, $\mathrm{R}=\mathrm{NH}_{2}, \mathrm{X}=\mathrm{COOEt}$

d, $\mathrm{R}=\mathrm{OH}, \mathrm{X}=\mathrm{COOEt}$

Sheme 2. Synthesis of compounds $8 \mathbf{a}, \mathbf{b} ; \mathbf{1 0}$ a,b and $\mathbf{1 1 a - d}$ 
<smiles>[X]c1c(N)sc2sc(-c3cc4ccccc4oc3=O)nc12</smiles>

$+$<smiles>CCOC(=O)CCC#N</smiles>

8a, $\mathrm{X}=\mathrm{CN}$

b, $\mathrm{X}=\mathrm{COOEt}$<smiles>[X]c1c(NC(=O)CN)sc2sc(-c3cc4ccccc4oc3=O)nc12</smiles>

12a, $\mathrm{X}=\mathrm{CN}$

b, $\mathrm{X}=\mathrm{COOE} t$
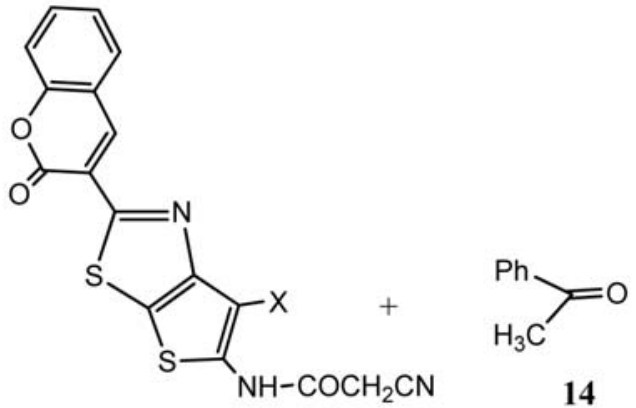

14

12a, $\mathrm{X}=\mathrm{CN}$

b, $\mathrm{X}=\mathrm{COOEt}$

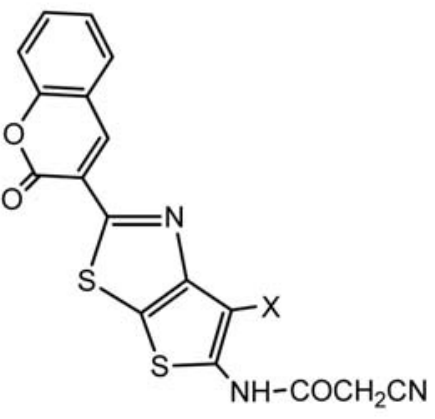

12a, $\mathrm{X}=\mathrm{CN}$

b, $\mathrm{X}=\mathrm{COOEt}$

13a, $\mathrm{R}=\mathrm{NH}_{2}$

b, $\mathrm{R}=\mathrm{OH}$

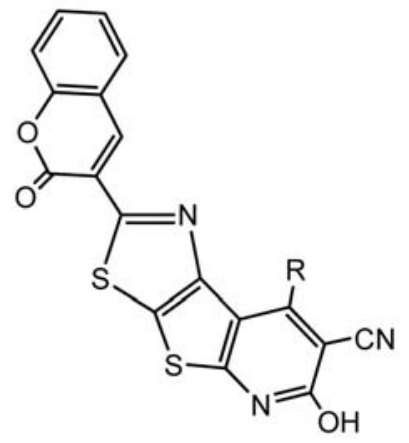

$\mathrm{NH}_{4} \mathrm{OAc}$

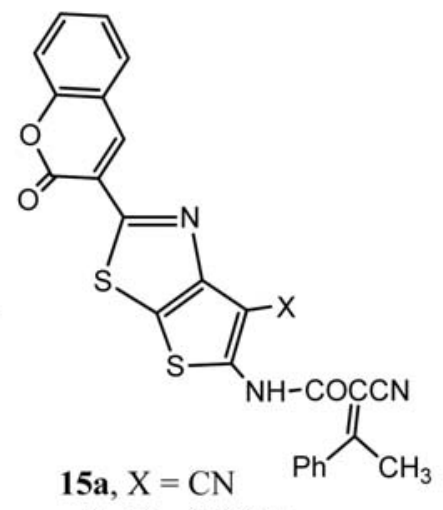

b, $X=$ COOEt

Sheme 3. Synthesis of compounds $12 \mathbf{a}, \mathbf{b} ; \mathbf{1 3} \mathbf{a}, \mathbf{b}$ and $\mathbf{1 5 a}, \mathbf{b}$

ting the coumarin $\mathrm{H}-4$ and a multiplet at $\delta 7.26-7.40 \mathrm{ppm}$ for the $\mathrm{C}_{6} \mathrm{H}_{4}$ group. In addition, the ${ }^{13} \mathrm{C}$ NMR spectrum signals were consistent with the assigned structure. The reaction of either compound $\mathbf{8 a}$ or $\mathbf{8 b}$ with phenylisothiocyanate (9) gave the 7-phenylthiazolo[ $\left[4^{\prime}, 5^{\prime}: 4,5\right]$ thieno[2,3- $d]$ pyrimidine-6(7H)-thione derivatives 10a and $10 b$, respectively. On the other hand, the reaction of either compound $\mathbf{8 a}$ or $\mathbf{8 b}$ with either malononitrile (4) or ethyl cyanoacetate $\left(\mathbf{4}^{\prime}\right)$ gave the 7-phenylthiazolo[ $\left.4^{\prime}, 5^{\prime}: 4,5\right]$ thieno[2,3- $d]$ pyridin-6(7H)-thione derivatives 11a-d, res- pectively (Scheme 2). The analytical and spectral data of the latter products were consistent with their respective structures (see experimental section).

The 2-amino group present in compounds 8a and $\mathbf{8 b}$ is capable for the amide group formation. Thus, the reaction of either $\mathbf{8 a}$ or $\mathbf{8 b}$ with ethyl cyanoacetate in refluxing dimethylformamide gave the amide derivatives 12a and 12b, respectively. The latter compounds readily underwent cyclization when heated in sodium ethoxide solution to give the 7-phenylthiazolo $\left[4^{\prime}, 5^{\prime}: 4,5\right]$ thieno 


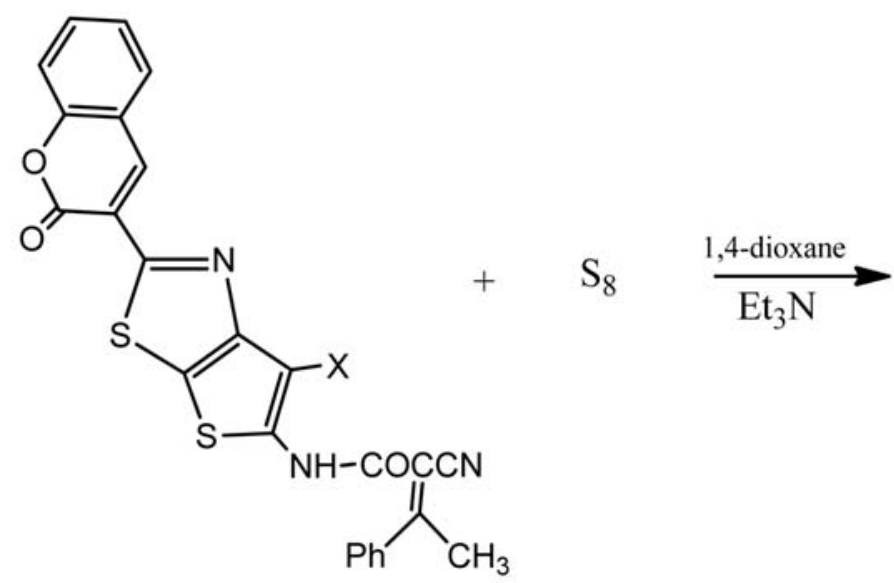

15a, $\mathrm{X}=\mathrm{CN}$

b, $X=$ COOEt
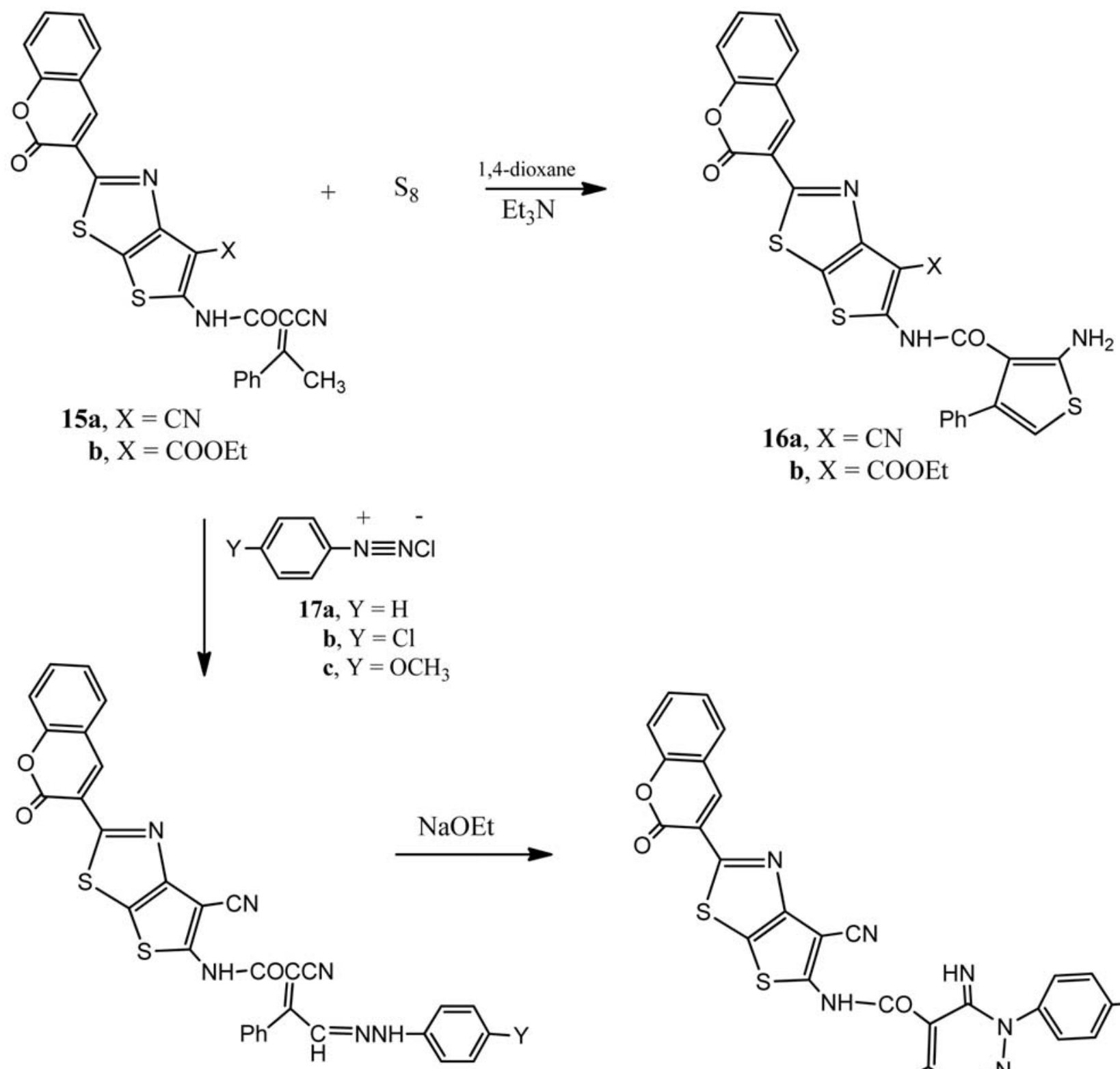

18a, $\mathrm{Y}=\mathrm{H}$

b, $\mathrm{Y}=\mathrm{Cl}$

c, $\mathrm{Y}=\mathrm{OCH}_{3}$

Sheme 4. Synthesis of compounds 16a, b; 18 a, $c$ and 19a-c

$[2,3-d]$ pyridine derivatives $\mathbf{1 3} \mathbf{a}$ and $\mathbf{1 3 b}$, respectively. On the other hand, the reaction of either compound 12a or 12b with acetophenone (14) gave the Knoevenagel condensation products $\mathbf{1 5 a}$ and $\mathbf{1 5} \mathbf{b}$, respectively (Scheme $3)$.

The presence of the but-2-enenitrile moiety in compounds 15a and 15b is suitable for thiophene synthesis. Thus, the reaction of either compound $\mathbf{1 5 a}$ or $\mathbf{1 5 b}$ with elemental sulfur gave the thiophene derivatives 16a and $\mathbf{1 6 b}$, respectively. On the other hand, the reaction of eit- her $15 a$ or $15 b$ with any of benzenediazonium chloride (17a), 4-chlorobenzenediazonium chloride (17b), or 4-methoxybenzenediazonium chloride $(\mathbf{1 7 c})$ gave the arylhydrazone derivatives $\mathbf{1 8 a}-\mathbf{c}$, respectively (Scheme 4). The spectral and analytical data of compounds 18a-c were in agreement with their respective structures (see experimental section). The latter compounds underwent cyclization when heated in sodium ethoxide solution to give corresponding pyridazine derivatives $19 \mathbf{a}-\mathbf{c}$, respectively. 


\section{1. Anti-inflammatory Evaluation Method}

Carrageenin-induced rat hind paw oedema model was used. The method adopted resembles essentially that described by Winter. ${ }^{19}$ The animals were studied for toxicity of DMSO up to $10 \% \mathrm{v} / \mathrm{v}$ in saline, and 5\% DMSO was selected as a vehicle to suspend the standard drugs and the test compounds.

Albino rats weighing between 150 and $250 \mathrm{~g}$ of either sex were starved for $18 \mathrm{~h}$ prior to the experiment. The animals were weighed, marked for identification and divided into groups of six. The standard drugs, ibuprofen (20 $\mathrm{mg} / \mathrm{kg}$ body weight), mefenamic acid (100 mg/kg body weight) and three graded doses (10, 20 and $40 \mathrm{mg} / \mathrm{kg}$ body weight) of the test compounds were given orally as a suspension using 5\% DMSO as a vehicle.

One hour later foot paw oedema was induced by injecting $0.1 \mathrm{~mL}$ of $1 \%$ carrageenin subcutaneously into the planter portion of the right hind paw of each rat. Initial foot paw volume was measured immediately by mercury plethysmometer. Oedema was measured $3 \mathrm{~h}$ after carrageenin administration. The swelling in test group animals was used to calculate the \% inhibition +/- SEM of oedema achieved by the compound at the test dose compared with the vehicle control group. The \% protection of oedema was calculated according to the formula:

$$
\% \text { anti inflammatory activity }=100\left(\frac{1-V_{t}}{V_{c}}\right)
$$

where $V_{t}$ and $V_{c}$ are the volume of oedema in test compounds and control groups, respectively.

\section{2. Anti-ulcer Evaluation Method}

Animals Wistar albino rats weighing 150-200 g of either sex maintained under standard husbandary conditions (temperature $23 \pm 2{ }^{\circ} \mathrm{C}$, relative humidity $55 \pm 10 \%$ and $12 \mathrm{~h} \mathrm{light/dark} \mathrm{cycle)} \mathrm{were} \mathrm{used} \mathrm{for} \mathrm{the} \mathrm{screening.}$ Animals were fed with standard laboratory food ad libitum during the study period.

Albino rats of either sex were divided into four groups of six animals each. Animals were fasted for $24 \mathrm{~h}$ before the study, but had free access to water. Animals in the control group received only distilled water. Each of the given compounds at 250 and $500 \mathrm{mg} / \mathrm{kg}$, (p. o.) were given to the animals in the treatment group. Ranitidine (50 $\mathrm{mg} / \mathrm{kg}$ ) was used as a standard. After $1 \mathrm{~h}$ of drugs treatment, they were anaesthetized with the help of anesthetic ether; the abdomen was opened by a small midline incision below the xiphoid process. Pyloric portion of the stomach was slightly lifted out and ligated according to the method of Shay ${ }^{20}$ avoiding traction to the pylorus or damage to its blood supply. The stomach was replaced carefully and the abdominal wall was closed by interrupted sutures. Rats were sacrificed by an over dose of anaesthetic ether after $4 \mathrm{~h}$ of pyloric ligation. The abdomen was opened, cardiac end of the stomach was dissected out and the contents were drained into a glass tube. The volume of the gastric juice was measured and centrifuged at 2000 $\mathrm{rpm}$ for $10 \mathrm{~min}$. From the supernatant, aliquots $(1 \mathrm{~mL}$ each) were taken for the determination of $\mathrm{pH}$, total and free acidity. Each stomach was examined for lesions in the fore stomach portion and indexed according to severity.

Determination of $\mathrm{pH}$ : an aliquot of $1 \mathrm{~mL}$ gastric juice was diluted with $1 \mathrm{~mL}$ of distilled water and $\mathrm{pH}$ of the solution was measured using $\mathrm{pH}$ meter.

\section{3. Toxicity Method on Shrimp Larvae}

All toxicity tests were 96-h static renewal tests and water quality measurements (dissolved oxygen, $\mathrm{pH}$, temperature, salinity) were taken in the control containers each day. Tests were run in a Revcos Environmental Chamber at $25{ }^{\circ} \mathrm{C}, 20 \%$ salinity, and a $16 \mathrm{~h}$ light $/ 8 \mathrm{~h}$ dark cycle. A media change was made every $24 \mathrm{~h}$. Larvae used for all tests were one to two days old and exposed in 600 $\mathrm{mL}$ glass beakers containing $400 \mathrm{~mL}$ of media with 10 larvae/beaker and three replicates/concentration. Larvae were fed newly hatched Artemia after daily media exchange. The concentration of each compound was taken in terms 10,100 and $100 \mathrm{mg} / \mathrm{mL}$. Adult shrimp toxicity tests were also run to complete the grass shrimp toxicity profile. Adult shrimp (acclimated for two weeks before testing) were exposed in $4 \mathrm{~L}$ wide mouth glass jars containing $2 \mathrm{~L}$ of media and $10 \mathrm{shrimp/jar}$ with two replicates/concentration, according to the modified method published by Delorenzo ${ }^{21}$ and were run under conditions as described above for larvae.

\section{4. Biological Evaluation}

\section{4. 1. Anti-inflammatory Evaluations}

From Table 1 it is clear that compounds $\mathbf{1 0 b}, \mathbf{1 3 b}$, 18a, 19b and 19c showed high anti-inflammatory activity, but compounds 6b, 6c, 7a, 8b, 11a, 11b, 13a, 16b and 18c showed low anti-inflammatory effect. Considering the 4,7-dihydrothiazolo[4,5-b]pyridine derivatives 7a-c, compound $\mathbf{7 b}$ with the 4-chlorophenyl moiety showed the highest anti-inflammatory activity among the three compounds. On the other hand, for the thieno[3,2- $d]$ thiazole derivatives $\mathbf{8 a}, \mathbf{b}$, compound $\mathbf{8 a}$ with the 3-cyano group showed higher anti-inflammatory activity than compound 8b with the COOEt moiety. For the thiazolo[4',5':4,5] thieno[2,3- $d$ ]pyrimidine derivatives 10a and 10b, it is obvious that compound $\mathbf{1 0 b}$ with the hydroxyl group showed higher anti-inflammatory activity than compound 10a with the amino group. Similarly for compounds 11a-d, compound 11d with the $\mathrm{OH}$ and COOEt moieties has the highest anti-inflammatory activity among the four compounds.

The reaction of either compound $\mathbf{8 a}$ or $\mathbf{8 b}$ with ethyl cyanoacetate gave the $N$-cyanoacetamido derivatives $\mathbf{1 2 a}$ 
or $\mathbf{1 2 b}$, respectively. Such changed showed a remarkable decrease of anti-inflammatory activity in the case of 12a. On the other hand, it showed an increase of anti-inflammatory activity of $\mathbf{1 2} \mathbf{b}$. For the thiazolo[ $\left.4^{\prime}, 5^{\prime}: 4,5\right]$ thieno[2,3-b]pyridine derivatives 13a,b the presence of the $\mathrm{OH}$ group in compound $\mathbf{1 3 b}$ is responsible for its higher anti-inflammatory activity than compound $\mathbf{1 3 a}$. The reaction of either compound 12a or $\mathbf{1 2 b}$ with acetophenone gave the condensation products $15 \mathbf{a}$ and $\mathbf{1 5} \mathbf{b}$, respectively, where compound $\mathbf{1 5 b}$ with the COOEt had higher activity than compound 15a with the $\mathrm{CN}$ group. However, the thiophene derivatives 16a,b obtained from 15a,b showed the reverse: compound $\mathbf{1 6 a}$ with the $\mathrm{CN}$ group showed higher anti-inflammatory activity than compound $\mathbf{1 6 b}$ with the COOEt moiety. Considering the arylhydrazone derivatives 18a-c, it is clear from Table 1 that compound 18a with the unsubstituted aryl moiety has the maximum anti-inflammatory activity among the three compounds. However, cyclization of compounds $\mathbf{1 8 a}-\mathbf{c}$ in sodium ethoxide solution gave the pyridazine derivatives 19a-c where compound $19 \mathrm{~b}$ with 4-chloroaryl group showed the highest anti-inflammatory activity among the three compounds, followed by the compound 19c with the 4-methoxyaryl group having the intermediate activity of the three compounds.

\section{5. Anti-ulcer Activity}

From Table 2 it is clear that compounds $\mathbf{6 a - c}, 7 \mathbf{a}-\mathbf{c}$, 8b, 10b, 13a, 16b, 19b and 19c showed the maximum antiulcer activity. Moreover, these twelve compounds showed inhibition effect higher than the reference drug ranitidine. On the other hand, most of the newly synthesized products showed a moderate anti-ulcer activity at concentrations 250 and $500 \mathrm{mg} / \mathrm{kg}$. Moreover, compounds $\mathbf{1 9 b}$ and 19c showed the maximum antiulcer activity among the tested compounds.

\section{5. 1. Macroscopic Evaluation of Stomach}

The stomachs were opened along the greater curvature, rinsed with saline to remove gastric contents and blood clots and examined by a $10 \times$ magnifier lens to assess the formation of ulcers. The numbers of ulcers were counted. Scoring of ulcer was made as follows: (0) $\cdots$ normal colored stomach; (0.5) $\cdots$ red coloration; (1) $\cdots$ spot ulcer; (1.5) $\cdots$ hemorrhagic streak; (2) $\cdots$ deep ulcers; (3) ... perforation. Mean ulcer score for each animal was expressed as ulcer index (UI). The percentage of ulcer protection was determined as follows. UI was measured by using the following formula:

$$
\mathrm{UI}=\mathrm{UN}+\mathrm{US}+\left(10^{-1} \mathrm{UP}\right)
$$

where UI is ulcer index; UN average number of ulcers per animal; US average number of severity score; UP percen- tage of animals with ulcers. Percentage inhibition of ulceration was calculated as below:

$$
\begin{aligned}
& \% \text { inhibition of ulceration }= \\
& \quad=[\mathrm{UI}(\text { control })-\mathrm{UI}(\text { text })] \frac{100}{\mathrm{UI}(\text { control })}
\end{aligned}
$$

\section{6. Toxicity}

Bioactive compounds are often toxic to shrimp larvae. Thus, in order to monitor these chemicals in vivo lethality to shrimp larvae (Artemia salina), brine shrimp lethality assay was used. Results were analyzed with $\mathrm{LC}_{50}$ program to determine $\mathrm{LC}_{50}$ values and $95 \%$ confidence intervals. ${ }^{22}$ Results are given in Table 4 for the compounds which exhibited optimal anti-inflammatory and anti-ulcer activity, that is the eleven compounds $\mathbf{7 b}$, 8a, 10a, 10b, 13a, 13b, 15b, 18a, 19b, 19c and 19d. The shrimp lethality assay is considered as a useful tool for preliminary assessment of toxicity, and it has been used for the detection of fungal toxins, plant extract toxicity, heavy metals, cyanobacteria toxins, pesticides, and cytotoxicity testing of dental materials, ${ }^{23}$ natural and synthetic organic compounds. ${ }^{24}$ It has also been shown that A. salina toxicity test results have a correlation with rodent and human acute oral toxicity data. Generally, a good correlation was obtained between A. salina toxicity test and the rodent data. Likewise, the predictive screening potential of the aquatic invertebrate tests for acute oral toxicity in humans, including A. salina toxicity test, was slightly better than the rat test for test compounds. ${ }^{25}$

In order to prevent the toxicity results from possible false effects originating from solubility of compounds and possible toxicity effect of DMSO, compounds were prepared by dissolving in DMSO in the suggested DMSO volume ranges. It is clear from Table 3 that compounds $\mathbf{1 0 b}$, 18a, 19c and 19d showed no toxicity against the tested organisms. On the other hand, compound $\mathbf{7 b}, \mathbf{1 3 b}$ and $\mathbf{1 5 b}$ are very toxic compounds, while the rest of compounds are harmful.

\section{Experimental}

\section{1. General}

All melting points were determined on an Electrothermal digital melting point apparatus and are uncorrected. IR spectra ( $\mathrm{KBr}$ discs) were recorded on a FTIR plus 460 or Pye Unicam SP-1000 spectrophotometer (Pye Unicam, UK, Cambridge). ${ }^{1} \mathrm{H}$ NMR and ${ }^{13} \mathrm{C}$ NMR spectra were recorded with Varian Gemini-200 (200 MHz, Varian UK) and JEOL AS $500 \mathrm{MHz}$ (JEOL, Japan) instruments in DMSO- $d_{6}$ as the solvent, using TMS as the internal standard, chemical shifts are expressed as $\delta \mathrm{ppm}$. The 
Table 1. Anti-inflammatory evaluation of the newly synthesis products

\begin{tabular}{|c|c|c|c|}
\hline \multirow[t]{2}{*}{$\begin{array}{r}\text { Compound } \\
\text { rat }\end{array}$} & \multicolumn{3}{|c|}{ Anti-inflammatory activity ${ }^{a}$ carrageenin-induced } \\
\hline & $10 \mathrm{mg} / \mathrm{kg}$ & $20 \mathrm{mg} / \mathrm{kg}$ & $40 \mathrm{mg} / \mathrm{kg}$ \\
\hline 3 & $0.60 \pm 0.04(74)$ & $0.38 \pm 0.06(83)$ & $0.19 \pm 0.07(92)$ \\
\hline $6 \mathbf{a}$ & $0.55 \pm 0.18(76)$ & $0.22 \pm 0.02(90)$ & $0.23 \pm 0.11(90)$ \\
\hline $6 \mathbf{b}$ & $0.99 \pm 0.23(57)$ & $0.68 \pm 0.08(70)$ & $1.13 \pm 0.38(51)$ \\
\hline 6c & $0.98 \pm 0.28(57)$ & $0.82 \pm 0.03(64)$ & $0.63 \pm 0.18(72)$ \\
\hline $7 \mathbf{a}$ & $0.82 \pm 0.15(64)$ & $0.60 \pm 0.13(73)$ & $0.92 \pm 0.18(58)$ \\
\hline $7 \mathbf{b}$ & $0.38 \pm 0.08(83)$ & $0.36 \pm 0.07(84)$ & $0.29 \pm 0.04(87)$ \\
\hline $7 c$ & $0.80 \pm 0.15(65)$ & $0.66 \pm 0.12(71)$ & $0.52 \pm 0.05(77)$ \\
\hline $8 \mathbf{a}$ & $0.44 \pm 0.08(81)$ & $0.51 \pm 0.09(78)$ & $0.05 \pm 0.04(98)$ \\
\hline $8 \mathbf{b}$ & $0.93 \pm 0.14(59)$ & $0.81 \pm 0.20(65)$ & $1.22 \pm 0.30(48)$ \\
\hline $10 \mathrm{a}$ & $1.30 \pm 0.04(43)$ & $0.48 \pm 0.06(79)$ & $0.93 \pm 0.14(59)$ \\
\hline $10 \mathrm{~b}$ & $0.09 \pm 0.02(96)$ & $0.16 \pm 0.08(93)$ & $0.19 \pm 0.06(92)$ \\
\hline $11 a$ & $1.22 \pm 0.18(47)$ & $1.09 \pm 0.23(53)$ & $1.19 \pm 0.37(48)$ \\
\hline 11b & $1.08 \pm 0.11(53)$ & $1.04 \pm 0.18(55)$ & $1.09 \pm 0.21(53)$ \\
\hline $11 \mathrm{c}$ & $0.83 \pm 0.17(64)$ & $0.92 \pm 0.20(60)$ & $0.69 \pm 0.30(70)$ \\
\hline 11d & $0.68 \pm 0.03(70)$ & $0.29 \pm 0.04(87)$ & $0.59 \pm 0.23(74)$ \\
\hline $12 \mathbf{a}$ & $0.83 \pm 0.19(64)$ & $1.29 \pm 0.36(44)$ & $1.33 \pm 0.46(42)$ \\
\hline $12 b$ & $0.60 \pm 0.10(74)$ & $0.88 \pm 0.08$ & $0.49 \pm 0.06(79)$ \\
\hline 13a & $1.54 \pm 0.20(33)$ & $1.01 \pm 0.23(56)$ & $0.51 \pm 0.13(78)$ \\
\hline $13 b$ & $0.39 \pm 0.02(83)$ & $0.09 \pm 0.01(96)$ & $0.39 \pm 0.13(83)$ \\
\hline $15 \mathbf{a}$ & $0.63 \pm 0.15(73)$ & $0.82 \pm 0.21(64)$ & $0.65 \pm 0.16(72)$ \\
\hline $15 b$ & $0.33 \pm 0.02(86)$ & $0.29 \pm 0.07(87)$ & $0.40 \pm 0.09(83)$ \\
\hline $16 \mathbf{a}$ & $0.75 \pm 0.19(67)$ & $0.32 \pm 0.04(86)$ & $0.22 \pm 0.06(90)$ \\
\hline $16 b$ & $1.39 \pm 0.21$ & $1.08 \pm 0.25(53)$ & $1.19 \pm 0.12(48)$ \\
\hline $18 \mathbf{a}$ & $0.13 \pm 0.07(94)$ & $0.24 \pm 0.04(89)$ & $0.07 \pm 0.03(97)$ \\
\hline $18 b$ & $0.73 \pm 0.18$ & $0.52 \pm 0.15(77)$ & $0.29 \pm 0.02(87)$ \\
\hline $18 c$ & $1.39 \pm 0.26(39)$ & $1.52 \pm 0.16(34)$ & $1.44 \pm 0.29(37)$ \\
\hline $19 a$ & $0.89 \pm 0.17(61)$ & $0.62 \pm 0.08(73)$ & $0.29 \pm 0.01(87)$ \\
\hline 19b & $0.18 \pm 0.03(92)$ & $0.18 \pm 0.09(92)$ & $0.09 \pm 0.01(96)$ \\
\hline $19 \mathrm{c}$ & $0.29 \pm 0.11(87)$ & $0.37 \pm 0.07(84)$ & $0.49 \pm 0.11(79)$ \\
\hline DMSO control & 2.30 & - & - \\
\hline Indomethacin & $0.32 \pm 0.09(86)$ & $0.31 \pm 0.07(88)$ & $0.09 \pm 0.29(96)$ \\
\hline
\end{tabular}

${ }^{a}$ Oral administration for all test compounds, $\mathrm{P}<0.05$, the standard drugs (dose and \% protection) were ibuprofen $(20 \mathrm{mg} / \mathrm{kg}, 33 \%)$ and mefenamic acid $(100 \mathrm{mg} / \mathrm{kg}, 39 \%)$.

mass spectra were recorded with Hewlett Packard 5988 A GC/MS system (Hewlett Packard, Agilent, USA) and GCMS-QP 1000Ex Shimadzu (EI, 70 eV) (Shimadzu, Japan) instruments. Analytical data were obtained from Vario EL III Elemental CHNS analyzer (Germany).

\section{1. 1. 2-(2-Oxo-2H-chromen-3-yl)thiazol-4(5H)- one (3)}

To a solution of compound $\mathbf{1}(1.40 \mathrm{~g}, 0.01 \mathrm{~mol})$ in 1,4-dioxane $(30 \mathrm{~mL})$ containing piperidine $(1.0 \mathrm{~mL}) \mathrm{sa}-$ licylaldehyde $(1.22 \mathrm{~g}, 0.01 \mathrm{~mol})$ was added. The reaction mixture, in each case, was heated under reflux for 1 $\mathrm{h}$, left to cool and the solid product formed, in each case, was collected by filtration and crystallized from ethanol.

Yellow crystals (ethanol), yield 82\% (2.45 g), mp 166-168 ${ }^{\circ} \mathrm{C}$; IR (KBr) vmax 3054, 2933, 1690, 1687, 1660, $1638 \mathrm{~cm}^{-1} ;{ }^{1} \mathrm{H}$ NMR (DMSO- $d_{6}, 200 \mathrm{MHz}$ ): $\delta$
7.28-7.38 (4H, m, Bz), $6.52(1 \mathrm{H}, \mathrm{s}, \mathrm{H}-4), 4.28(2 \mathrm{H}, \mathrm{s}$, $\mathrm{CH}_{2}$ ); ${ }^{13} \mathrm{C}$ NMR (DMSO- $d_{6}, 75 \mathrm{MHz}$ ): $\delta 170.3$ (C-2'), 168.6, 164.3 (C-2, C-4'), 58.6 (C-5'), 144.1, 139.5, 133.8, 130.1, 126.3, 123.6, 120.3 (C-3, C-4, C-5, C-6, C-7, C-8, C-9, C-10); EI-MS: $m / z 245[\mathrm{M}]^{+}(18)$; Analysis Calcd for $\mathrm{C}_{12} \mathrm{H}_{7} \mathrm{NO}_{3} \mathrm{~S}(245.25): \mathrm{C}, 58.77 ; \mathrm{H}, 2.88 ; \mathrm{N}, 5.71 ; \mathrm{S}$, $13.07 \%$. Found: C, $58.85 ; \mathrm{H}, 3.03 ; \mathrm{N}, 5.92 ; \mathrm{S}, 12.89 \%$.

\section{1. 2. General Procedure for the Synthesis of the Pyrano[2,3- $d]$ thiazole Derivatives 6a-c}

To a solution of compound $3(2.45 \mathrm{~g}, 0.01 \mathrm{~mol})$ in ethanol $(50 \mathrm{~mL})$ containing triethylamine $(0.50 \mathrm{~mL}) \mathrm{ma}-$ lononitrile $(0.66 \mathrm{~g}, 0.01 \mathrm{~mol})$ and any of benzaldehyde $(1.06 \mathrm{~g}, 0.01 \mathrm{~mol}), 4$-chlorobenzaldehyde $(1.40 \mathrm{~g}, 0.01$ mol) or 4-methoxybenzaldehyde $(1.37 \mathrm{~g}, 0.01 \mathrm{~mol})$ were added. The whole reaction mixture was heated under reflux for $4 \mathrm{~h}$ then left to cool. The formed solid product, in each case, was collected by filtration. 
Table 2. Effect of DMSO solution of the given compounds on gastric ulcer induced by pylorus ligation in rats, $\mathrm{pH}$, total and free acidity in pyloric ligation induced ulceration in rats

\begin{tabular}{|c|c|c|c|}
\hline Treatment & $\begin{array}{c}\text { Dose } \\
(\mathrm{mg} / \mathrm{kg})\end{array}$ & $\begin{array}{l}\text { Ulcer } \\
\text { index }\end{array}$ & $\begin{array}{c}\% \text { ulcer } \\
\text { inhibition }\end{array}$ \\
\hline $\begin{array}{l}\text { Control } \\
\text { (distilled } \\
\text { water) }\end{array}$ & 10 & $3.6 \pm 0.45$ & - \\
\hline \multirow[t]{2}{*}{3} & 250 & $1.91 \pm 0.53$ & 47 \\
\hline & 500 & $0.98 \pm 0.19$ & 73 \\
\hline \multirow[t]{2}{*}{$6 \mathbf{a}$} & 250 & $0.43 \pm 0.08$ & 88 \\
\hline & 500 & $0.18 \pm 0.01$ & 95 \\
\hline \multirow[t]{2}{*}{$6 \mathbf{b}$} & 250 & $0.32 \pm 0.28$ & 91 \\
\hline & 500 & $0.82 \pm 0.08$ & 77 \\
\hline \multirow[t]{2}{*}{$6 c$} & 250 & $0.77 \pm 0.19$ & 79 \\
\hline & 500 & $0.59 \pm 0.08$ & 84 \\
\hline \multirow[t]{2}{*}{$7 \mathbf{a}$} & 250 & $0.66 \pm 0.42$ & 82 \\
\hline & 500 & $0.49 \pm 0.52$ & 86 \\
\hline \multirow[t]{2}{*}{$7 b$} & 250 & $0.88 \pm 0.30$ & 75 \\
\hline & 500 & $0.38 \pm 021$ & 89 \\
\hline \multirow[t]{2}{*}{ 7c } & 250 & $0.58 \pm 0.27$ & 84 \\
\hline & 500 & $0.32 \pm 0.19$ & 91 \\
\hline \multirow[t]{2}{*}{$8 \mathbf{a}$} & 250 & $1.29 \pm 0.27$ & 64 \\
\hline & 500 & $1.38 \pm 0.36$ & 62 \\
\hline \multirow[t]{2}{*}{$8 b$} & 250 & $0.13 \pm 0.08$ & 96 \\
\hline & 500 & $0.19 \pm 0.05$ & 95 \\
\hline \multirow[t]{2}{*}{$10 a$} & 250 & $1.86 \pm 0.63$ & 48 \\
\hline & 500 & $0.82 \pm 0.17$ & 77 \\
\hline \multirow[t]{2}{*}{$10 b$} & 250 & $0.80 \pm 0.25$ & 78 \\
\hline & 500 & $0.32 \pm 0.09$ & 91 \\
\hline \multirow[t]{2}{*}{$11 \mathrm{a}$} & 250 & $1.77 \pm 0.83$ & 51 \\
\hline & 500 & $1.20 \pm 0.39$ & 67 \\
\hline \multirow[t]{2}{*}{ 11b } & 250 & $1.83 \pm 0.29$ & 49 \\
\hline & 500 & $0.66 \pm 0.13$ & 82 \\
\hline \multirow[t]{2}{*}{$11 \mathrm{c}$} & 250 & $1.73 \pm 0.42$ & 52 \\
\hline & 500 & $1.39 \pm 0.62$ & 61 \\
\hline \multirow[t]{2}{*}{ 11d } & 250 & $1.23 \pm 0.64$ & 66 \\
\hline & 500 & $0.88 \pm 0.12$ & 75 \\
\hline \multirow[t]{2}{*}{$12 \mathbf{a}$} & 250 & $1.93 \pm 0.25$ & 46 \\
\hline & 500 & $0.83 \pm 0.26$ & 77 \\
\hline \multirow[t]{2}{*}{$12 b$} & 250 & $1.82 \pm 0.63$ & 49 \\
\hline & 500 & $1.94 \pm 0.08$ & 46 \\
\hline \multirow[t]{2}{*}{$13 \mathbf{a}$} & 250 & $0.63 \pm 0.09$ & 82 \\
\hline & 500 & $1.19 \pm 0.16$ & 67 \\
\hline \multirow[t]{2}{*}{ 13b } & 250 & $1.09 \pm 0.15$ & 70 \\
\hline & 500 & $0.86 \pm 0.26$ & 76 \\
\hline \multirow[t]{2}{*}{$15 a$} & 250 & $1.73 \pm 0.41$ & 52 \\
\hline & 500 & $1.63 \pm 0.22$ & 55 \\
\hline $15 b$ & 250 & $1.87 \pm 0.48$ & 48 \\
\hline & 500 & $1.29 \pm 0.27$ & 64 \\
\hline $16 a$ & 250 & $1.53 \pm 0.42$ & 57 \\
\hline & 500 & $0.89 \pm 0.04$ & 75 \\
\hline $16 b$ & 250 & $0.63 \pm 0.12$ & 82 \\
\hline & 500 & $0.58 \pm 0.20$ & 84 \\
\hline $18 \mathbf{a}$ & 250 & $0.92 \pm 0.13$ & 66 \\
\hline & 500 & $0.62 \pm 0.23$ & 74 \\
\hline $18 b$ & 250 & $2.88 \pm 0.53$ & 20 \\
\hline & 500 & $2.21 \pm 0.09$ & 39 \\
\hline $18 \mathrm{c}$ & 250 & $1.42 \pm 0.96$ & 60 \\
\hline & 500 & $0.89 \pm 0.25$ & 72 \\
\hline
\end{tabular}

\begin{tabular}{lccc}
\hline Treatment & $\begin{array}{c}\text { Dose } \\
(\mathrm{mg} / \mathrm{kg})\end{array}$ & $\begin{array}{c}\text { Ulcer } \\
\text { index }\end{array}$ & $\begin{array}{c}\text { \% ulcer } \\
\text { inhibition }\end{array}$ \\
\hline 19a & 250 & $2.89 \pm 0.68$ & 20 \\
& 500 & $1.63 \pm 0.71$ & 55 \\
19b & 250 & $0.22 \pm 0.05$ & 94 \\
& 500 & $0.29 \pm 0.09$ & 92 \\
19c & 250 & $0.26 \pm 0.06$ & 93 \\
& 500 & $0.19 \pm 0.01$ & 95 \\
\hline Ranitidine & $\mathbf{5 0}$ & $\mathbf{1 . 6 5} \pm \mathbf{0 . 4 9}$ & $\mathbf{5 4}$ \\
\hline
\end{tabular}

Values are expressed as mean \pm S.E.M., $n=6, * p<0.05$ when compared with control group.

Table 3. Toxicity of the most potent compounds

\begin{tabular}{|c|c|c|c|c|c|c|}
\hline $\begin{array}{l}\text { Com- } \\
\text { pound }\end{array}$ & $\begin{array}{r}\text { Conc. } \\
(\mu \mathrm{g} / \\
\mathrm{mL})\end{array}$ & $\begin{array}{c}\text { Morta- } \\
\text { lity }^{a}\end{array}$ & Toxicity & $\mathbf{L C}_{\mathbf{5 0}}$ & $\begin{array}{c}\text { Upper } \\
95 \% \\
\text { lim. }\end{array}$ & $\begin{array}{c}\text { Lower } \\
95 \% \\
\text { lim. }\end{array}$ \\
\hline \multirow[t]{3}{*}{$\overline{7 b}$} & 10 & 2 & very toxic & 120.29 & - & - \\
\hline & 100 & 4 & & & & \\
\hline & 1000 & 10 & & & & \\
\hline \multirow{3}{*}{$\begin{array}{l}\mathbf{8 a} \\
148.38\end{array}$} & 10 & 1 & harmful & 210.55 & 197.22 & \\
\hline & 100 & 4 & & & & \\
\hline & 1000 & 7 & & & & \\
\hline \multirow[t]{3}{*}{$10 \mathrm{~b}$} & 10 & 0 & nontoxic & 818.15 & 112.40 & 72.73 \\
\hline & 100 & 2 & & & & \\
\hline & 1000 & 4 & & & & \\
\hline \multirow[t]{3}{*}{$13 b$} & 10 & 3 & very toxic & 112.49 & 276.40 & 66.30 \\
\hline & 100 & 6 & & & & \\
\hline & 1000 & 10 & & & & \\
\hline \multirow[t]{3}{*}{$15 b$} & 10 & 2 & very toxic & 109.06 & 220.31 & 80.45 \\
\hline & 100 & 5 & & & & \\
\hline & 1000 & 10 & & & & \\
\hline \multirow[t]{3}{*}{$18 \mathbf{a}$} & 10 & 0 & non toxic & 909.28 & - & - \\
\hline & 100 & 1 & & & & \\
\hline & 1000 & 4 & & & & \\
\hline \multirow[t]{3}{*}{$19 b$} & 10 & 1 & harmful & 133.40 & 236.50 & 93.28 \\
\hline & 100 & 4 & & & & \\
\hline & 1000 & 10 & & & & \\
\hline \multirow[t]{3}{*}{$19 c$} & 10 & 0 & non toxic & 910.63 & - & - \\
\hline & 100 & 3 & & & & \\
\hline & 1000 & 5 & & & & \\
\hline \multirow[t]{3}{*}{ 19d } & 10 & 0 & non toxic & 890.63 & - & - \\
\hline & 100 & 2 & & & & \\
\hline & 1000 & 6 & & & & \\
\hline
\end{tabular}

${ }^{\text {a }}$ Ten organisms (A. salina) tested for each concentration.

5-Amino-2-(2-oxo-2H-chromen-3-yl)-7-phenyl-7Hpyrano[2,3-d]thiazole-6-carbonitrile $(6 a)$

Yellow crystals (ethanol), yield $80 \%$ (3.19 g), mp 188-191 ${ }^{\circ} \mathrm{C}$; IR (KBr) vmax 3477, 3329, 3056, 2220, 1693, 1654, $1635 \mathrm{~cm}^{-1}$; ${ }^{1} \mathrm{H}$ NMR (DMSO- $d_{6}, 200 \mathrm{MHz}$ ): 反 7.42-7.25 (9H, m, 2Bz), $6.58\left(1 \mathrm{H}, \mathrm{s}, \mathrm{H}-4^{\prime}\right), 6.18(1 \mathrm{H}, \mathrm{s}$, $\mathrm{H}-7), 4.82\left(2 \mathrm{H}, \mathrm{s}, \mathrm{D}_{2} \mathrm{O}\right.$ exchangeable, $\left.\mathrm{NH}_{2}\right) ;{ }^{13} \mathrm{C}$ NMR (DMSO- $d_{6}, 75 \mathrm{MHz}$ ): $\delta 86.4(\mathrm{C}-7), 116.8(\mathrm{CN}), 144.4$, $143.1,142.8,138.8,135.2,130.3,129.4,128.9,127.2$, 
126.3, 126.2, 125.4, 124.8, 122.8, 119.8 (Bz, C-8,C-9, C-5, C-6, C-1', C-2', C3', C-4', C-5', C-6'), 165.2 (C-7'), 173.2 (C-2); EI-MS: $m / z 399$ [M] ${ }^{+}(42 \%)$; Analysis Calcd for $\mathrm{C}_{22} \mathrm{H}_{13} \mathrm{~N}_{3} \mathrm{O}_{3} \mathrm{~S}$ (399.42): C, 66.15; H, 3.28; N, 10.52; $\mathrm{S}$, 8.03. Found: C, 66.29; H, 3.41; N, 10.73; S, 7.92.

5-Amino-7-(4-chlorophenyl)-2-(2-oxo-2H-chromen-3yl)-7H-pyrano[2,3- $d]$ thiazole-6-carbonitrile $(6 \mathrm{~b})$

Pale yellow crystals (ethanol), yield 73\% (3.16 g), $\mathrm{mp} 166-169{ }^{\circ} \mathrm{C}$; IR (KBr) vmax 3493, 3326, 3054, 2223,1690, 1656, $1633(\mathrm{C}=\mathrm{C}) \mathrm{cm}^{-1} ;{ }^{1} \mathrm{H}$ NMR (DMSO- $d_{6}$, $200 \mathrm{MHz}): \delta 7.24-7.40(8 \mathrm{H}, \mathrm{m}, 2 \mathrm{Bz}), 6.61\left(1 \mathrm{H}, \mathrm{s}, \mathrm{H}-4^{\prime}\right)$, $6.15(1 \mathrm{H}, \mathrm{s}, \mathrm{H}-7), 4.80$ (s, 2H, $\mathrm{D}_{2} \mathrm{O}$ exchangeable, $\mathrm{NH}_{2}$ ); ${ }^{13} \mathrm{C}$ NMR (DMSO- $\left.d_{6}, 75 \mathrm{MHz}\right): \delta 86.8(\mathrm{C}-7), 116.6(\mathrm{CN})$, $120.4,122.6,123.9,124.3,124.9,125.2,125.7,126.9$, $129.2,130.5,133.9,135.2,138.1,140.2,142.9,143.6$ (Bz, C-8,C-9,C-5, C-6, C-1', C-2', C3', C-4', C-5', C-6'), 165.4 (C-7'), 173.0 (C-2); EI-MS: $m / z 433$ [M] ${ }^{+}(30 \%)$; Analysis Calcd for $\mathrm{C}_{22} \mathrm{H}_{12} \mathrm{ClN}_{3} \mathrm{O}_{3} \mathrm{~S}$ (433.03): C, 60.90; H, 2.79 ; N, 9.69; S, 7.39\%. Found: C, 61.28; H, 2.91; N, $9.49 ; \mathrm{S}, 7.44 \%$.

5-Amino-7-(4-methoxyphenyl)-2-(2-oxo-2H-chromen3-yl)-7H-pyrano[2,3- $d]$ thiazole-6-carbonitrile (6c)

Orange crystals (ethanol), yield $88 \%$ (3.77 g), mp 211-213 ${ }^{\circ} \mathrm{C}$; IR (KBr) vmax $3475,3318,3056,2221$, 1692, 1658,1630 $\mathrm{cm}^{-1} ;{ }^{1} \mathrm{H}$ NMR (DMSO- $\left.d_{6}, 200 \mathrm{MHz}\right): \delta$ 7.29-7.41 (m, 8H, 2Bz), $6.63\left(1 \mathrm{H}, \mathrm{s}, \mathrm{H}-4{ }^{\prime}\right), 6.18(1 \mathrm{H}, \mathrm{s}$, $\mathrm{H}-7), 4.86\left(2 \mathrm{H}, \mathrm{s}, \mathrm{D}_{2} \mathrm{O}\right.$ exchangeable, $\left.\mathrm{NH}_{2}\right), 3.14(3 \mathrm{H}, \mathrm{s}$, $\left.\mathrm{OCH}_{3}\right) ;{ }^{13} \mathrm{C}$ NMR (DMSO- $\left.d_{6}, 75 \mathrm{MHz}\right): \delta 173.3(\mathrm{C}-2)$, 165.4 (C-7'), 143.6, 143.0, 139.2, 138.7, 134.6, 133.7, 132.2, 131.8, 129.8, 127.4, 126.0, 125.7, 125.1, 124.6, 123.2, 120.7 (Bz, C-8,C-9,C-5, C-6, C-1', C-2', C3', C-4', C-5', C-6'), 116.8 (CN), 86.6 (C-7), 34.6 (C, $\mathrm{OCH}_{3}$ ); EI-MS: $m / z 429[\mathrm{M}]^{+}(22 \%)$; Analysis Calcd for $\mathrm{C}_{23} \mathrm{H}_{15} \mathrm{~N}_{3} \mathrm{O}_{4} \mathrm{~S}$ (429.45): C, 64.33; H, 3.52; N, 9.78; S, 7.47\%. Found: C, 64.40; H, 3.66; N, 9.83; S, 7.54\%.

\section{1. 3. General procedure for the synthesis of the pyridino $[2,3-d]$ thiazole derivatives $7 \mathbf{a}-\mathrm{c}$}

To a solution of compound $3(2.45 \mathrm{~g}, 0.01 \mathrm{~mol})$ in ethanol $(50 \mathrm{~mL})$ containing ammonium acetate $(0.50 \mathrm{~mL})$ malononitrile $(0.66 \mathrm{~g}, 0.01 \mathrm{~mol})$ and any of benzaldehyde $(1.06 \mathrm{~g}, 0.01 \mathrm{~mol}), 4$-chlorobenzaldehyde $(1.40 \mathrm{~g}, 0.01$ mol) or 4-methoxybenzaldehyde $(1.37 \mathrm{~g}, 0.01 \mathrm{~mol})$ was added. The whole reaction mixture was heated under reflux for $4 \mathrm{~h}$ then left to cool. The solid product formed upon pouring onto ice/water containing a few drops of hydrochloric acid was collected by filtration.

\section{5-Amino-2-(2-oxo-2H-chromen-3-yl)-7-phenyl-4,7- dihydrothiazolo[4,5-b]pyridine-6-carbonitrile (7a)}

Yellow crystals (ethanol), yield 77\% (3.06 g), mp 201-203 ${ }^{\circ} \mathrm{C}$; IR (KBr) vmax 3466-3380, 3059, 2222, $1688,1652,1633 \mathrm{~cm}^{-1} ;{ }^{1} \mathrm{H}$ NMR (DMSO- $d_{6}, 200 \mathrm{MHz}$ ): $\delta 8.23\left(\mathrm{~s}, 1 \mathrm{H}, \mathrm{D}_{2} \mathrm{O}\right.$ exchangeable, $\left.\mathrm{NH}\right), 7.28-7.39(\mathrm{~m}, 9 \mathrm{H}$, 2Bz), 6.54 (1H, s, H-7), 6.16 (1H, s, H-4'), 4.80 (s, 2H, $\mathrm{D}_{2} \mathrm{O}$ exchangeable, $\mathrm{NH}_{2}$ ); ${ }^{13} \mathrm{C}$ NMR (DMSO- $d_{6}, 75$ MHz): $\delta 173.0$ (C-2), 165.8 (C-7'), 142.6, 141.3, 140.2, 139.4, 136.2, 134.9, 133.8, 128.7, 128.2, 126.8, 126.0, 125.3, 124.5, 121.5, 120.3 (Bz, C-8,C-9,C-5, C-6, C-1', C-2', C3', C-4', C-5', C-6'), 116.9 (CN), 86.2 (C-7); EI-MS: $m / z \quad 398\left[\mathrm{M}^{+}\right.$(28\%); Analysis Calcd for $\mathrm{C}_{22} \mathrm{H}_{14} \mathrm{~N}_{4} \mathrm{O}_{2} \mathrm{~S}$ (398.44): C, 66.32; H, 3.54; N, 14.06; $\mathrm{S}$, $8.05 \%$. Found: C, 66.59; H, 3.33; N, 14.18; S, 7.98\%.

5-Amino-7-(4-chlorophenyl)-2-(2-oxo-2H-chromen-3yl)-4,7-dihydrothiazolo[4,5-b]pyridine-6-carbonitrile (7b)

Pale yellow crystals (ethanol), yield 73\% (3.15 g), mp 241-243 ${ }^{\circ} \mathrm{C}$; IR (KBr) vmax 3493-3327, 3056, 2220, $1688,1653,1630 \mathrm{~cm}^{-1}$; ${ }^{1} \mathrm{H}$ NMR (DMSO- $d_{6}, 200 \mathrm{MHz}$ ): $\delta 8.25\left(\mathrm{~s}, 1 \mathrm{H}, \mathrm{D}_{2} \mathrm{O}\right.$ exchangeable, $\left.\mathrm{NH}\right), 7.26-7.39(\mathrm{~m}, 8 \mathrm{H}$, 2Bz), 6.63 (1H, s, H-7), 6.18 (1H, s, H-4'), 4.83 (s, 2H, $\mathrm{D}_{2} \mathrm{O}$ exchangeable, $\mathrm{NH}_{2}$ ); ${ }^{13} \mathrm{C}$ NMR (DMSO- $d_{6}, 75$ MHz): $\delta 173.2$ (C-2), 165.6 (C-7'), 142.8, 141.4, 140.8, 137.2, 135.6, 134.3, 132.8, 128.7, 126.4, 126.0, 125.2, 123.9, 123.2, 122.4, 120.3 (Bz, C-8,C-9,C-5, C-6, C-1', C-2', C3', C-4', C-5', C-6'), 116.9 (CN), 86.2 (C-7); EI-MS: $m / z \quad 432[\mathrm{M}]^{+}$(38\%); Analysis Calcd for $\mathrm{C}_{22} \mathrm{H}_{13} \mathrm{ClN}_{4} \mathrm{O}_{2} \mathrm{~S}$ (432.88): C, 61.04; H, 3.03; N, 12.94; S, 7.41\%. Found: C, 61.29; H, 2.88; N, 12.72; S, 7.39\%.

5-Amino-7-(4-methoxyphenyl)-2-(2-oxo-2H-chromen3-yl)-4,7-dihydrothiazolo[4,5-b]pyridine-6-carbonitrile $(7 \mathrm{c})$

Orange crystals (ethanol), yield 79\% (3.38 g), mp 255-258 ${ }^{\circ} \mathrm{C}$; IR (KBr) vmax 3488-3359, 3052, 2220, $1689,1654,1632 \mathrm{~cm}^{-1} ;{ }^{1} \mathrm{H}$ NMR (DMSO- $d_{6}, 200 \mathrm{MHz}$ ): $\delta 8.22\left(\mathrm{~s}, 1 \mathrm{H}, \mathrm{D}_{2} \mathrm{O}\right.$ exchangeable, $\left.\mathrm{NH}\right), 7.25-7.45(8 \mathrm{H}, \mathrm{m}$, 2Bz), $6.62(1 \mathrm{H}, \mathrm{s}, \mathrm{H}-7), 6.20(1 \mathrm{H}, \mathrm{s}, \mathrm{H}-4$ '), 4.83 (s, 2H, $\mathrm{D}_{2} \mathrm{O}$ exchangeable, $\left.\mathrm{NH}_{2}\right), 3.70\left(\mathrm{~s}, 3 \mathrm{H}, \mathrm{CH}_{3}\right) ;{ }^{13} \mathrm{C}$ NMR (DMSO- $\left.d_{6}, 75 \mathrm{MHz}\right): \delta 173.2$ (C-2), 165.6 (C-7'), 142.9, $142.8,139.9,139.1,136.0,134.2,132.8,132.4,130.2$, $128.8,126.2,125.6,125.2,123.8,122.0,121.2(\mathrm{Bz}$, C-8, C-9, C-5, C-6, C-1', C-2', C3', C-4', C-5', C-6'), $116.9(\mathrm{CN}), 86.6(\mathrm{C}-7), 34.8\left(\mathrm{C}, \mathrm{OCH}_{3}\right)$, EI-MS: $m / z 428$ $[\mathrm{M}]^{+}(20 \%)$; Analysis Calcd for $\mathrm{C}_{23} \mathrm{H}_{16} \mathrm{~N}_{4} \mathrm{O}_{3} \mathrm{~S}$ (428.46): C, 64.47; H, 3.76; N, 13.08; S, 7.48\%. Found: C, 64.53; H, $3.80 ; \mathrm{N}, 12.93 ; \mathrm{S}, 7.62 \%$.

\section{1. 4. General Procedure for the Synthesis of the Thieno[3,2- $d]$ thiazole Derivatives 8a and 8b}

To a solution of compound $3(2.45 \mathrm{~g}, 0.01 \mathrm{~mol})$ in 1,4-dioxane $(40 \mathrm{~mL})$ containing $(0.01 \mathrm{~mol})$ triethylamine, either malononitrile $(0.66 \mathrm{~g}, 0.01 \mathrm{~mol})$ or ethyl cyanoacetate $(1.13 \mathrm{~g}, 0.01 \mathrm{~mol})$ and elemental sulfur $(0.32 \mathrm{~g}, 0.01$ mol) were added. The whole reaction mixture was heated under reflux for $4 \mathrm{~h}$ then left to cool. The solid product 
formed upon pouring onto ice/water containing a few drops of hydrochloric acid was collected by filtration.

\section{5-Amino-2-(2-oxo-2H-chromen-3-yl)thieno[3,2-d] thia- zole-6-carbonitrile (8a)}

Orange crystals (1,4-dioxane), yield 82\% (2.66 g), mp 189-193 ${ }^{\circ} \mathrm{C}$; IR (KBr) vmax 3488-3342, 3057, 2220, $1690,1655,1632 \mathrm{~cm}^{-1}$; ${ }^{1} \mathrm{H}$ NMR (DMSO- $d_{6}, 200 \mathrm{MHz}$ ): $\delta$ 7.40-7.26 (4H, m, Bz), $6.62(1 \mathrm{H}, \mathrm{s}, \mathrm{H}-4$ '), $4.93(2 \mathrm{H}, \mathrm{s}$, $\mathrm{D}_{2} \mathrm{O}$ exchangeable, $\mathrm{NH}_{2}$ ); ${ }^{13} \mathrm{C}$ NMR (DMSO- $d_{6}, 75$ MHz): $\delta 172.8$ (C-2), 164.8 (C-2'), 142.3, 141.8, 138.1, 136.2, 133.2, 132.8, 130.5, 128.4, 126.2, 123.9, 122.0, 119.7 (Bz, C-4, C-5, C-7, C-8, C-3', C-4'), $117.3(\mathrm{CN})$; EI-MS: $\mathrm{m} / \mathrm{z} 325[\mathrm{M}]^{+}$(15\%); Analysis Calcd for $\mathrm{C}_{15} \mathrm{H}_{7} \mathrm{~N}_{3} \mathrm{O}_{2} \mathrm{~S}_{2}$ (325.36): C, 55.37; H, 2.17; N, 12.91; S, $19.71 \%$. Found: C, 55.52; H, 2.28; N, 13.18; S, $19.88 \%$.

Ethyl 5-amino-2-(2-oxo-2H-chromen-3-yl)thieno[3,2d]thiazole-6-carboxylate (8b) Yellow crystals (1,4-dioxane), yield $77 \%$ (3.86 g), mp $142-145{ }^{\circ} \mathrm{C}$; IR (KBr) vmax 3493-3338, 3055, 1688, 1653, $1630 \mathrm{~cm}^{-1} ;{ }^{1} \mathrm{H}$ NMR (DMSO- $\left.d_{6}, 200 \mathrm{MHz}\right): \delta 7.38-7.23(4 \mathrm{H}, \mathrm{m}, \mathrm{Bz}), 6.64$ (1H, s, H-4'), $4.96\left(2 \mathrm{H}, \mathrm{s}, \mathrm{D}_{2} \mathrm{O}\right.$ exchangeable, $\left.\mathrm{NH}_{2}\right), 4.20$ $\left(2 \mathrm{H}, \mathrm{q}, J=7.29 \mathrm{~Hz}, \mathrm{OCH}_{2} \mathrm{CH}_{3}\right), 1.14(3 \mathrm{H}, \mathrm{t}, J=7.29 \mathrm{~Hz}$, $\mathrm{OCH}_{2} \mathrm{CH}_{3}$ ); ${ }^{13} \mathrm{C}$ NMR (DMSO- $d_{6}, 75 \mathrm{MHz}$ ): $\delta 172.9$ (C-2), 164.8 (C-2'), 142.6, 141.2, 138.3, 136.0, 133.6, $132.5,130.3,128.3,126.4,123.7,122.2,119.3$ (Bz, C-4, C-5, C-7, C-8, C-3', C-4'), $56.2\left(\mathrm{C}, \mathrm{CH}_{2}, \mathrm{OCH}_{2} \mathrm{CH}_{3}\right)$, $16.9\left(\mathrm{C}, \mathrm{CH}_{3}, \mathrm{OCH}_{2} \mathrm{CH}_{3}\right)$; EI-MS: $m / z, 372[\mathrm{M}]^{+}(30 \%)$; Analysis Calcd for $\mathrm{C}_{17} \mathrm{H}_{12} \mathrm{~N}_{2} \mathrm{O}_{4} \mathrm{~S}_{2}$ (372.42): C, 54.83; H, 3.25 ; N, 7.52; S, 17.22\%. Found: C, 54.76; H, 3.19; N, $7.73 ; \mathrm{S}, 17.08 \%$.

\section{1. 5. General Procedure for the Synthesis of 7- phenylthiazolo $\left[4^{\prime}, 5^{\prime}: 4,5\right]$-thieno $[2,3-d]$ pyrimidine-6(7H)-thione Derivatives 10 a and 10b}

To a solution of either compound $8 \mathbf{a}(3.25 \mathrm{~g}, 0.01$ $\mathrm{mol})$ or $\mathbf{8 b}$ (3.72 g, $0.01 \mathrm{~mol})$ in 1,4-dioxane (40 mL) containing triethylamine $(0.50 \mathrm{~mL})$, phenylisothiocyanate $(1.30 \mathrm{~g}, 0.01 \mathrm{~mol})$ was added. The whole reaction mixture was heated under reflux for $4 \mathrm{~h}$ then poured onto ice/water containing a few drops of hydrochloric acid and the formed solid product, in each case, was collected by filtration.

3-(8-Amino-7-phenyl-6-thioxo-6,7-dihydrothiazolo $\left[4^{\prime}, 5 ': 4,5\right]$ thieno[2,3- $\left.d\right]$ pyrimidin-2-yl)-2H-chromen-2one (10a)

Pale yellow crystals (1,4-dioxane), 74\% (3.40 g), mp 222-225 ${ }^{\circ} \mathrm{C}$; IR (KBr) vmax 3459-3326, 3054, 2223, 1687, 1653, 1630, $1220 \mathrm{~cm}^{-1}$; ${ }^{1} \mathrm{H}$ NMR (DMSO- $d_{6}, 200$ MHz): $\delta 7.25-7.42(\mathrm{~m}, 9 \mathrm{H}, 2 \mathrm{Bz}), 6.62\left(1 \mathrm{H}, \mathrm{s}, \mathrm{H}-4^{\prime}\right), 4.92$ $\left(2 \mathrm{H}, \mathrm{s}, \mathrm{D}_{2} \mathrm{O}\right.$ exchangeable, $\left.\mathrm{NH}_{2}\right) ;{ }^{13} \mathrm{C}$ NMR (DMSO- $d_{6}$, $75 \mathrm{MHz}): \delta 178.2$ (C-6), 170.8, 172.4 (C-2, C-10), 164.3
(C-2'), 142.6, 140.4, 139.2, 138.1, 136.2, 133.2, 132.8, $131.8,130.2$, 129.3, 128.6, 127.3, 125.8, 123.3, 121.8, 120.3 (2Bz, C-4, C-8, C-9, C-11, C-3', C-4'); EI-MS: $\mathrm{m} / \mathrm{z}$ $460[\mathrm{M}]^{+}$(35\%); Analysis Calcd for $\mathrm{C}_{22} \mathrm{H}_{12} \mathrm{~N}_{4} \mathrm{O}_{2} \mathrm{~S}_{3}$ (460.55): C, 57.37; H, 2.63; N, 12.17; S, 20.89\%. Found: C, 57.50; H, 2.58; N, 12.22; S, 20.69\%.

3-(8-Hydroxy-7-phenyl-6-thioxo-6,7-dihydrothiazolo[4',5':4,5]thieno[2,3- $d]$ pyrimidin-2-yl)-2H-chromen2-one (10b)

Yellow crystals (1,4-dioxane), yield 69\% (3.18 g), mp 180-184 ${ }^{\circ} \mathrm{C}$; IR (KBr) vmax 3473-3340, 3052, 2220, $1689,1652,1632,1223 \mathrm{~cm}^{-1} ;{ }^{1} \mathrm{H}$ NMR (DMSO- $d_{6}, 200$ MHz): $\delta 10.31\left(1 \mathrm{H}, \mathrm{s}, \mathrm{D}_{2} \mathrm{O}\right.$ exchangeable, $\left.\mathrm{OH}\right), 7.46-7.28$ (9H, m, 2Bz), 6.64 (1H, s, H-4'); ${ }^{13} \mathrm{C}$ NMR (DMSO- $d_{6}, 75$ MHz): $\delta 178.2$ (C-6), 170.5, 172.2 (C-2, C-10), 164.4 (C-2'), 143.1, 140.6, 138.0, 137.4, 136.1, 134.9, 132.6, 132.3, 130.6, 129.0, 126.1, 124.2, 123.6, 122.8, 120.5, 118.5 (2Bz, C-4, C-8, C-9, C-11, C-3', C-4'); EI-MS: $\mathrm{m} / \mathrm{z}$ $462[\mathrm{M}]^{+}$(18\%); Analysis Calcd for $\mathrm{C}_{22} \mathrm{H}_{11} \mathrm{~N}_{3} \mathrm{O}_{3} \mathrm{~S}_{3}$ (462.54): C, 57.25; H, 2.40; N, 9.10; S, 20.84\%. Found: C, 57.39; H, 2.29; N, 9.28; S, 20.77\%.

\section{1.6. General Procedure for the Synthesis of the Thiazolo $\left[4^{\prime}, 5\right.$ ':4,5] thieno[2,3-b]pyridine Derivatives 11a-d}

To a solution of either compound $8 \mathbf{a}$ (3.25 g, 0.01 $\mathrm{mol})$ or $8 \mathbf{b}(4.61 \mathrm{~g}, 0.01 \mathrm{~mol})$ in 1,4-dioxane $(30 \mathrm{~mL})$ containing triethylamine $(0.50 \mathrm{~mL})$, either malononitrile $(0.66 \mathrm{~g}, 0.01 \mathrm{~mol})$ or ethyl cyanoacetate $(1.13 \mathrm{~g}, 0.01 \mathrm{~mol})$ was added. The reaction mixture was heated under reflux for $2 \mathrm{~h}$ then poured onto ice/water containing a few drops of hydrochloric acid and the solid product formed was collected by filtration.

6,8-Diamino-2-(2-oxo-2H-chromen-3-yl)thiazolo [4',5':4,5]thieno[2,3-b]pyridine-7-carbonitrile (11a)

Orange crystals (1,4-dioxane), yield 78\% (4.83 g), $\mathrm{mp} 243-246^{\circ} \mathrm{C}$; IR (KBr) vmax 3488-3336, 3056, 2221 , $1689,1653,1632 \mathrm{~cm}^{-1} ;{ }^{1} \mathrm{H}$ NMR (DMSO- $d_{6}, 200 \mathrm{MHz}$ ): $\delta$ 7.40-7.28 (4H, m, Bz), $6.65\left(1 \mathrm{H}, \mathrm{s}, \mathrm{H}-4{ }^{\prime}\right), 5.21,4.89$ $\left(4 \mathrm{H}, 2 \mathrm{~s}, \mathrm{D}_{2} \mathrm{O}\right.$ exchangeable, $\left.2 \mathrm{NH}_{2}\right) ;{ }^{13} \mathrm{C}$ NMR (DMSO- $d_{6}$, $75 \mathrm{MHz}$ ): $\delta 171.8,170.3$ (C-2, C-6), 164.6 (C-2'), 140.5, 138.2, 136.4, 135.2, 134.6, 132.7, 130.2, 129.7, 127.8, 125.0, 124.6, 122.7, 120.1, 119.2 (Bz, C-4, C-5, C-8, C-9, C-10, C-11, C-3', C-4'), 116.9 (CN); EI-MS: $m / z 391$ [M] ${ }^{+}$ (24\%); Analysis Calcd for $\mathrm{C}_{18} \mathrm{H}_{9} \mathrm{~N}_{5} \mathrm{O}_{2} \mathrm{~S}_{2}$ (391.43): C, 55.23; H, 2.32; N, 17.89; S, 16.38\%. Found: C, 55.36; H, $2.41 ; \mathrm{N}, 18.29 ; \mathrm{S}, 16.29 \%$.

6-Amino-8-hydroxy-2-(2-oxo-2H-chromen-3-yl)thiazolo $\left[4^{\prime}, 5\right.$ ': 4,5$]$ thieno $[2,3-b]$ pyridine-7-carbonitrile (11b)

Pale yellow crystals (1,4-dioxane), yield 66\% (2.58 g), mp 210-213 ${ }^{\circ} \mathrm{C}$; IR (KBr) vmax 3574-3332, 3054, 
2222, 1686, 1651, $1631 \mathrm{~cm}^{-1}$; ${ }^{1} \mathrm{H}$ NMR (DMSO- $d_{6}, 200$ $\mathrm{MHz}): \delta 10.30\left(1 \mathrm{H}, \mathrm{s}, \mathrm{D}_{2} \mathrm{O}\right.$ exchangeable, $\left.\mathrm{OH}\right), 7.25-7.36$ $(4 \mathrm{H}, \mathrm{m}, \mathrm{Bz}), 6.63\left(1 \mathrm{H}, \mathrm{s}, \mathrm{H}-4^{\prime}\right), 4.87$ (2H, s, $\mathrm{D}_{2} \mathrm{O}$ exchangeable, $\mathrm{NH}_{2}$ ); ${ }^{13} \mathrm{C}$ NMR (DMSO- $d_{6}, 75 \mathrm{MHz}$ ): $\delta 171.5$, 170.2 (C-2, C-6), 164.3 (C-2'), 141.8, 137.0, 136.4, 133.0, 132.8, 131.9, 130.2, 125.7, 125.1, 124.6, 123.4, 121.8, 120.6 (Bz, C-4, C-5, C-8, C-9, C-10, C-11, C-3', C-4'),116.5 (CN); EI-MS: m/z $392[\mathrm{M}]^{+}(18 \%)$; Analysis Calcd for $\mathrm{C}_{18} \mathrm{H}_{8} \mathrm{~N}_{4} \mathrm{O}_{3} \mathrm{~S}_{2}$ (392.41): C, 55.09; H, 2.05; N, 14.28; S, 16.34\%. Found: C, 55.18; H, 2.21; N, 14.50; S, $16.48 \%$.

Ethyl 6,8-diamino-2-(2-oxo-2H-chromen-3-yl)thiazolo $\left[4^{\prime}, 5\right.$ ':4,5] thieno[2,3-b]-pyridine-7-carboxylate (11c)

Yellowish white (1,4-dioxane), yield 73\% (3.19 g), mp $188-192{ }^{\circ} \mathrm{C}$; IR (KBr) vmax 3464-3328, 3056, 1688, $1653,1629 \mathrm{~cm}^{-1} ;{ }^{1} \mathrm{H}$ NMR (DMSO- $d_{6}, 200 \mathrm{MHz}$ ): $\delta$ 7.37-7.27 (4H, m, Bz), $6.60(1 \mathrm{H}, \mathrm{s}, \mathrm{H}-4$ '), 4.45, $5.28(4 \mathrm{H}$, $2 \mathrm{~s}, \mathrm{D}_{2} \mathrm{O}$ exchangeable, $\left.2 \mathrm{NH}_{2}\right), 4.20(2 \mathrm{H}, \mathrm{q}, J=7.08 \mathrm{~Hz}$, $\left.\mathrm{OCH}_{2} \mathrm{CH}_{3}\right), 1.14\left(3 \mathrm{H}, \mathrm{t}, J=7.08 \mathrm{~Hz}, \mathrm{OCH}_{2} \mathrm{CH}_{3}\right) ;{ }^{13} \mathrm{C}$ NMR (DMSO- $\left.d_{6}, 75 \mathrm{MHz}\right): \delta 170.4,171.4$ (C-2, C-6), 164.2 (C-2'), 120.5, 121.8, 121.9, 122.8, 123.9, 124.2, 127.3, 127.8, 128.0, 129.3, 130.3, 131.3, 134.9, 139.2 (Bz, C-4, C-5, C-8, C-9, C-10, C-11, C-3', C-4'), 54.2 (C, $\left.\mathrm{CH}_{2}, \mathrm{OCH}_{2} \mathrm{CH}_{3}\right), 16.8\left(\mathrm{C}, \mathrm{CH}_{3}, \mathrm{OCH}_{2} \mathrm{CH}_{3}\right)$; EI-MS: $\mathrm{m} / \mathrm{z}$ $438[\mathrm{M}]^{+}(23 \%)$; Analysis Calcd for $\mathrm{C}_{20} \mathrm{H}_{14} \mathrm{~N}_{4} \mathrm{O}_{4} \mathrm{~S}_{2}$ (438.48): C, 54.78; H, 3.22; N, 12.78; S, 14.63\%. Found: C, 54.91; H, 3.40; N, 12.91; S, 14.80\%.

Ethyl 6-amino-8-hydroxy-2-(2-oxo-2H-chromen-3-yl) thiazolo $\left[4^{\prime}, 5\right.$ ': 4,5$]$ thieno $[2,3-b]$ pyridine-7-carboxylate (11d)

Yellow crystals (1,4-dioxane), yield 78\% (3.42 g), mp 205-208 ${ }^{\circ} \mathrm{C}$; IR (KBr) vmax 3520-3336, 3058, 1689, $1651,1632 \mathrm{~cm}^{-1} ;{ }^{1} \mathrm{H}$ NMR (DMSO- $d_{6}, 200 \mathrm{MHz}$ ): $\delta$ $10.22\left(1 \mathrm{H}, \mathrm{s}, \mathrm{D}_{2} \mathrm{O}\right.$ exchangeable, $\left.\mathrm{OH}\right), 7.28-7.39(4 \mathrm{H}, \mathrm{m}$, Bz), $6.62\left(1 \mathrm{H}, \mathrm{s}, \mathrm{H}-4\right.$ '), $4.48\left(2 \mathrm{H}, \mathrm{s}, \mathrm{D}_{2} \mathrm{O}\right.$ exchangeable, $\left.\mathrm{NH}_{2}\right), 4.20\left(2 \mathrm{H}, \mathrm{q}, J=7.22 \mathrm{~Hz}, \mathrm{OCH}_{2} \mathrm{CH}_{3}\right), 1.15(3 \mathrm{H}, \mathrm{t}, J$ $\left.=7.22 \mathrm{~Hz}, \mathrm{OCH}_{2} \mathrm{CH}_{3}\right) ;{ }^{13} \mathrm{C}$ NMR $\left(\mathrm{DMSO}-d_{6}, 75 \mathrm{MHz}\right): \delta$ 171.6, 170.2 (C-2, C-6), 164.4 164.2 (C-2'), 142.7, 134.9, 132.6, 131.8, 131.2, 129.7, 119.3, 126.7, 126.5, 124.8, 124.0, 123.6, 122.8, 121.6, (Bz, C-4, C-5, C-8, C-9, C-10, C-11, C-3', C-4'), 54.6 (C, $\left.\mathrm{CH}_{2}, \mathrm{OCH}_{2} \mathrm{CH}_{3}\right), 16.7$ (C, $\mathrm{CH}_{3}, \mathrm{OCH}_{2} \mathrm{CH}_{3}$ ); EI-MS: $\mathrm{m} / z 439[\mathrm{M}]^{+}(18 \%)$; Analysis Calcd for $\mathrm{C}_{20} \mathrm{H}_{13} \mathrm{~N}_{3} \mathrm{O}_{5} \mathrm{~S}_{2}$ (439.46): $\mathrm{C}, 54.66 ; \mathrm{H}, 2.98 ; \mathrm{N}$, 9.56; S, 14.59\%. Found: C, 54.79; H, 3.17; N, 9.29; S, $14.63 \%$.

\section{1. 7. General Procedure for the Synthesis of the 2-cyanoacetylthieno[3,2-d]thiazole Deriva- tives $12 \mathrm{a}$ and $12 \mathrm{~b}$}

To a solution of either compound 8a (3.25 g, 0.01 $\mathrm{mol})$ or $\mathbf{8 b}$ (4.61 g, $0.01 \mathrm{~mol})$ in dimethylformamide (30 $\mathrm{mL})$ ethyl cyanoacetate $(1.13 \mathrm{~g}, 0.01 \mathrm{~mol})$ was added. The reaction mixture was heated under reflux for $3 \mathrm{~h}$ then pou- red onto ice/water containing a few drops of hydrochloric acid and the solid product formed was collected by filtration.

2-Cyano- $N$-[6-cyano-2-(2-oxo-2H-chromen-3-yl)thieno[3,2- $d]$ thiazol-5-yl]acetamide (12a)

Yellow crystals (1,4-dioxane), yield 83\% (3.25 g), mp 188-191 ${ }^{\circ} \mathrm{C}$; IR (KBr) vmax 3468-3326, 3054, 2223, $2220,1688,1705,1655,1634 \mathrm{~cm}^{-1} ;{ }^{1} \mathrm{H}$ NMR (DMSO- $d_{6}$, $200 \mathrm{MHz}): \delta 8.20\left(1 \mathrm{H}, \mathrm{s}, \mathrm{NH}, \mathrm{D}_{2} \mathrm{O}\right.$ exchangeable), 7.26-7.36 (4H, m, Bz), $6.63(1 \mathrm{H}, \mathrm{s}, \mathrm{H}-4$ '), $5.30(2 \mathrm{H}, \mathrm{s}$, $\left.\mathrm{CH}_{2}\right) ;{ }^{13} \mathrm{C}$ NMR (DMSO- $d_{6}, 75 \mathrm{MHz}$ ): $\delta 170.1(\mathrm{C}-2)$, 164.4, 165.2 (C-2', C, CO, $\mathrm{COCH}_{2}$ ), 141.2, 134.9, 134.8, 132.9, 131.9, 128.3, 126.9, 125.3, 124.7, 122.3, 121.9, 120.8 (Bz, C-4, C-5, C-7, C-8, C-3', C-4'), 116.6, 115.8 $(2 \mathrm{CN}), 62.5\left(\mathrm{C}, \mathrm{CH}_{2}, \mathrm{COCH}_{2}\right)$; EI-MS: $\mathrm{m} / z, 392[\mathrm{M}]^{+}$ (18\%); Analysis Calcd for $\mathrm{C}_{18} \mathrm{H}_{8} \mathrm{~N}_{4} \mathrm{O}_{3} \mathrm{~S}_{2}$ (392.41): C, 55.09; H, 2.05; N, 14.28; S, 16.34\%. Found: C, 55.28; H, $2.26 ; \mathrm{N}, 14.25 ; \mathrm{S}, 16.52 \%$.

Ethyl 5-(2-cyanoacetamido)-2-(2-oxo-2H-chromen-3yl)thieno[3,2-d] thiazole-6-carboxylate (12b)

Pale brown crystals (1,4-dioxane), yield 80\% (3.51 g), mp 166-169 ${ }^{\circ} \mathrm{C}$; IR (KBr) vmax 3462-3329, 3057, 2222, 1689-1706, 1646, $1628 \mathrm{~cm}^{-1} ;{ }^{1} \mathrm{H}$ NMR (DMSO- $d_{6}$, $200 \mathrm{MHz}): \delta 8.31\left(1 \mathrm{H}, \mathrm{s}, \mathrm{D}_{2} \mathrm{O}\right.$ exchangeable, $\left.\mathrm{NH}\right)$, 7.39-7.26 (4H, m, Bz), $6.61(1 \mathrm{H}, \mathrm{s}, \mathrm{H}-4$ '), $5.28(2 \mathrm{H}, \mathrm{s}$, $\left.\mathrm{CH}_{2}\right), 4.22\left(2 \mathrm{H}, \mathrm{q}, J=6.83 \mathrm{~Hz}, \mathrm{OCH}_{2} \mathrm{CH}_{3}\right), 1.38(3 \mathrm{H}, \mathrm{t}, J$ $\left.=6.83 \mathrm{~Hz}, \mathrm{OCH}_{2} \mathrm{CH}_{3}\right) ;{ }^{13} \mathrm{C} \mathrm{NMR}\left(\mathrm{DMSO}-d_{6}, 75 \mathrm{MHz}\right): \delta$ 170.0 (C-2), 162.3, 163.0, 164.4 (C-2', COOEt, NHCO), $140.3,134.8,131.9,128.4,126.9,126.0,125.3,124.1$, 123.1, 122.2, 121.3, 120.8 (Bz, C-4, C-5, C-7, C-8, C-3', C-4'), $116.8(\mathrm{CN}), 62.8\left(\mathrm{C}, \mathrm{CH}_{2}, \mathrm{COCH}_{2}\right), 54.2(\mathrm{C}$, $\left.\mathrm{OCH}_{2} \mathrm{CH}_{3}\right), 16.5\left(\mathrm{C}, \mathrm{OCH}_{2} \mathrm{CH}_{3}\right)$; EI-MS: $m / 2,439[\mathrm{M}]^{+}$ (37\%); Analysis Calcd for $\mathrm{C}_{20} \mathrm{H}_{13} \mathrm{~N}_{3} \mathrm{O}_{5} \mathrm{~S}_{2}$ (439.46): $\mathrm{C}$, 54.66; H, 2.98; N, 9.56; S, 14.59\%. Found: C, 54.80; H, $3.11 ; \mathrm{N}, 9.73 ; \mathrm{S}, 14.79 \%$.

\section{1. 8. General Procedure for the Synthesis of the Thiazolo[4',5':4,5] thieno[ $[2,3-b]$ pyridine- 7-carbonitrile Derivatives 13a and 13b}

To a suspension of either compound 12a (3.92 g, $0.01 \mathrm{~mol})$ or $\mathbf{1 2 b}(4.39 \mathrm{~g}, 0.01 \mathrm{~mol})$ in sodium ethoxide solution [prepared by dissolving metallic sodium $(0.46 \mathrm{~g}$, $0.02 \mathrm{~mol})$ in absolute ethanol $(40 \mathrm{~mL})]$ was heated in a boiling water bath for $3 \mathrm{~h}$ then poured onto ice/water containing a few drops of hydrochloric acid (till $\mathrm{pH}$ 6). The formed solid product, in each case, was collected by filtration.

8-Amino-6-hydroxy-2-(2-oxo-2H-chromen-3-yl)thiazolo[4',5':4,5] thieno[2,3-b]pyridine-7-carbonitrile (13a)

Yellow crystals (1,4-dioxane), yield 73\% (2.86 g), mp 245-247 ${ }^{\circ} \mathrm{C}$; IR (KBr) vmax 3533-3341, 3058, 2220, 
1693, 1653, $1632 \mathrm{~cm}^{-1} ;{ }^{1} \mathrm{H}$ NMR (DMSO- $d_{6}, 200 \mathrm{MHz}$ ): $\delta$ $10.19\left(1 \mathrm{H}, \mathrm{s}, \mathrm{OH}, \mathrm{D}_{2} \mathrm{O}\right.$ exchangeable), 7.39-7.28 (4H, m, $\mathrm{Bz}), 6.60\left(1 \mathrm{H}, \mathrm{s}, \mathrm{H}-4^{\prime}\right), 4.80\left(2 \mathrm{H}, \mathrm{s}, \mathrm{D}_{2} \mathrm{O}\right.$ exchangeable, $\mathrm{NH}_{2}$ ); ${ }^{13} \mathrm{C}$ NMR (DMSO- $d_{6}, 75 \mathrm{MHz}$ ): $\delta 171.8,170.5$ (C-2, C-6), 164.2 (C-2'), 139.8, 137.9, 132.4, 131.9, 130.3, 129.7, 127.8, 127.1, 124.9, 124.2, 123.8, 122.3, 121.6, 120.2 (Bz, C-4, C-5, C-8, C-9, C-10, C-11, C-3', C-4'), $116.8(\mathrm{CN})$; EI-MS: $\mathrm{m} / z 392[\mathrm{M}]^{+}(32 \%)$; Analysis Calcd for $\mathrm{C}_{18} \mathrm{H}_{8} \mathrm{~N}_{4} \mathrm{O}_{3} \mathrm{~S}_{2}$ (392.41): $\mathrm{C}, 55.09 ; \mathrm{H}, 2.05 ; \mathrm{N}, 14.28 ; \mathrm{S}$, $16.34 \%$. Found: C, 54.89; H, 2.31; N, 14.44; S, $16.41 \%$.

\section{6,8-Dihydroxy-2-(2-oxo-2H-chromen-3-yl)thiazolo} $\left[4^{\prime}, 5 ': 4,5\right]$ thieno[2,3-b]pyridine-7-carbonitrile (13b)

Yellow crystals (1,4-dioxane), yield 73\% (2.86 g), mp 262-265 ${ }^{\circ} \mathrm{C}$; IR (KBr) vmax 3542-3318, 3054, 1686, $1642,1628 \mathrm{~cm}^{-1} ;{ }^{1} \mathrm{H}$ NMR (DMSO- $d_{6}, 200 \mathrm{MHz}$ ): $\delta$ $10.32,10.11\left(2 \mathrm{H}, 2 \mathrm{~s}, \mathrm{D}_{2} \mathrm{O}\right.$ exchangeble, 2OH), 7.36-7.28 $\left(4 \mathrm{H}, \mathrm{m}, \mathrm{C}_{6} \mathrm{H}_{4}\right), 6.64\left(1 \mathrm{H}, \mathrm{s}, \mathrm{H}-4\right.$ '); ${ }^{13} \mathrm{C}$ NMR (DMSO- $d_{6}$, $75 \mathrm{MHz}): \delta 171.0,170.2$ (C-2, C-6), 164.2 (C-2'), 142.0, $138.3,130.9,130.6,129.4,128.3,127.3,126.3,125.8$, 125.4, 125.0, 123.9, 123.5, 122.6, 122.0, 120.2 (Bz, C-4, C-5, C-8, C-9, C-10, C-11, C-3', C-4'), 116.6 (CN); EI-MS: $\mathrm{m} / z 393[\mathrm{M}]^{+}$(44\%); Analysis Calcd for $\mathrm{C}_{18} \mathrm{H}_{7} \mathrm{~N}_{3} \mathrm{O}_{4} \mathrm{~S}_{2}$ (393.40): C, 54.96; H, 1.79; N, 10.68; S, $16.30 \%$. Found: C, 54.77; H, 2.01; N, 10.29; S, $16.42 \%$.

\section{1. 9. General Procedure for the Synthesis of the Thieno[3,2- $d]$ thiazole Derivatives 15a and 15b}

To a dry solid of either 12a $(3.92 \mathrm{~g}, 0.01 \mathrm{~mol})$ or $\mathbf{1 2 b}$ $(4.39 \mathrm{~g}, 0.01 \mathrm{~mol})$ acetophenone $(1.20 \mathrm{~g}, 0.01 \mathrm{~mol})$ and ammonium acetate $(0.50 \mathrm{~g})$ was added. The whole reaction mixture was heated in an oil bath at $120{ }^{\circ} \mathrm{C}$ for $0.5 \mathrm{~h}$ and the solid product formed upon trituration with ethanol was collected by filtration.

2-Cyano- $N$-[6-cyano-2-(2-oxo-2H-chromen-3-yl)thieno[3,2-d] thiazol-5-yl]-3-phenylbut-2-enamide (15a)

Yellow crystals (1,4-dioxane), yield 73\% (3.60 g), mp 221-223 ${ }^{\circ} \mathrm{C}$; IR (KBr) vmax 3472-3318, 3057, 2222, 2220, 1689, 1690, 1642, $1627 \mathrm{~cm}^{-1}$; ${ }^{1} \mathrm{H}$ NMR (DMSO- $d_{6}$, $200 \mathrm{MHz}): \delta 10.22\left(1 \mathrm{H}, \mathrm{s}, \mathrm{D}_{2} \mathrm{O}\right.$ exchangeable, $\left.\mathrm{NH}\right)$, 7.26-7.46 (9H, m, 2Bz), $6.59(1 \mathrm{H}, \mathrm{s}, \mathrm{H}-4$ '), $2.88(3 \mathrm{H}, \mathrm{s}$, $\mathrm{CH}_{3}$ ); ${ }^{13} \mathrm{C}$ NMR (DMSO- $d_{6}, 75 \mathrm{MHz}$ ): $\delta 170.2(\mathrm{C}-2)$, 164.5, 162.8 (C-2, NHCO), 140.5, 138.7, 136.2, 133.4, 132.6, 132.1, 130.8, 128.9, 127.8, 127.4, 126.1, 125.6, $124.2,123.7,122.1,121.9,120.9,120.5$ (2Bz, C-3, C-4, C-6, C-7, C-3', C-4'), 117.0,116.4, (2CN), 90.6, 86.7 $(\mathrm{C}=\mathrm{C}), 19.5\left(\mathrm{C}, \mathrm{CH}_{3}\right)$; EI-MS: $m / z 494[\mathrm{M}]^{+}(23 \%)$; Analysis Calcd for $\mathrm{C}_{26} \mathrm{H}_{14} \mathrm{~N}_{4} \mathrm{O}_{3} \mathrm{~S}_{2}$ (494.54): C, 63.14; H, 2.85; N, 11.33; S, 12.97\%. Found: C, 62.98; H, 2.69; N, $11.41 ; \mathrm{S}, 13.08 \%$.

Ethyl 2-\{[6-cyano-2-(2-oxo-2H-chromen-3-yl)thieno[3,2d] thiazol-5-yl]carbamoyl\}-3-phenylbut-2-enoate (15b)
Yellow crystals (1,4-dioxane), yield 68\% (3.67 g), mp 189-192 ${ }^{\circ} \mathrm{C}$; IR (KBr) vmax 3488-3326, 3052, 2221, 1690, 1682, 1638, $1628 \mathrm{~cm}^{-1}$; ${ }^{1} \mathrm{H}$ NMR (DMSO- $d_{6}, 200$ $\mathrm{MHz}): \delta 8.31\left(1 \mathrm{H}, \mathrm{s}, \mathrm{D}_{2} \mathrm{O}\right.$ exchangeble, $\left.\mathrm{NH}\right), 7.39-7.26$ $\left(9 \mathrm{H}, \mathrm{m}, \mathrm{C}_{6} \mathrm{H}_{5}, \mathrm{C}_{6} \mathrm{H}_{4}\right), 6.63(1 \mathrm{H}, \mathrm{s}, \mathrm{H}-4$ '), $4.22(\mathrm{q}, 2 \mathrm{H}, J=$ $\left.6.88 \mathrm{~Hz}, \mathrm{OCH}_{2} \mathrm{CH}_{3}\right), 2.68\left(3 \mathrm{H}, \mathrm{s}, \mathrm{CH}_{3}\right), 1.13(\mathrm{t}, 3 \mathrm{H}, J=$ $6.88 \mathrm{~Hz}, \mathrm{OCH}_{2} \mathrm{CH}_{3}$ ); ${ }^{13} \mathrm{C}$ NMR (DMSO- $d_{6}, 75 \mathrm{MHz}$ ): $\delta$ 170.4 (C-2), 164.2 (C-2'), 163.9, 162.3 (C-2, NHCO), 141.2, 134.7, 132.6, 130.8, 129.2, 128.3, 127.1, 126.8, $125.8,124.9,124.2,123.9,123.5,123.1,122.2,119.6$ (2Bz, C-3, C-4, C-6, C-7, C-3', C-4'), 90.8, 86.3 (C=C), $52.3\left(\mathrm{C}, \mathrm{OCH}_{2} \mathrm{CH}_{3}\right), 19.2\left(\mathrm{CH}_{3}\right), 16.8\left(\mathrm{C}, \mathrm{OCH}_{2} \mathrm{CH}_{3}\right)$, EI-MS: $m / z \quad 541\left[^{[\mathrm{M}]^{+}}(28 \%)\right.$; Analysis Calcd for $\mathrm{C}_{28} \mathrm{H}_{19} \mathrm{~N}_{3} \mathrm{O}_{5} \mathrm{~S}_{2}$ (541.60): C, 62.09; H, 3.54; N, 7.76; S, $11.84 \%$. Found: C, 61.95; H, 3.72; N, 8.02; S, $12.03 \%$.

\section{1. 10. General Procedure for the Synthesis of Thiophene Derivatives 16a and 16b}

To a solution of either compound 15a (4.94 g, 0.01 $\mathrm{mol})$ or $15 \mathrm{~b}(5.41 \mathrm{~g}, 0.01 \mathrm{~mol})$ in 1,4-dioxane $(30 \mathrm{~mL})$ containing triethylamine $(0.50 \mathrm{~mL})$ elemental sulfur $(3.2$ $\mathrm{g}, 0.01 \mathrm{~mol}$ ) was added. The reaction mixture was heated under reflux for $2 \mathrm{~h}$ then poured onto ice/water containing a few drops of hydrochloric acid and the solid product formed, in each case, was collected by filtration.

\section{2-Amino- $N$-[6-cyano-2-(2-oxo-2H-chromen-3-yl)thie- no[3,2-d] thiazol-5-yl]-4-phenylthiophene-3-carboxa- mide (16a)}

Yellow crystals (acetic acid), 82\% (4.31 g), mp 187-190 ${ }^{\circ} \mathrm{C}$; IR (KBr) vmax 3494-3326, 3053, 2224,1687, 1694, 1646, $1629 \mathrm{~cm}^{-1} ;{ }^{1} \mathrm{H}$ NMR (DMSO- $d_{6}$, $200 \mathrm{MHz}): \delta 10.28\left(1 \mathrm{H}, \mathrm{s}, \mathrm{D}_{2} \mathrm{O}\right.$ exchangeable, $\left.\mathrm{NH}\right)$, 7.23-7.39 (9H, m, 2Bz), $6.61(1 \mathrm{H}, \mathrm{s}, \mathrm{H}-4$ ') $6.24(1 \mathrm{H}, \mathrm{s}$, $\mathrm{H}-5$ "), $4.68\left(2 \mathrm{H}, \mathrm{s}, \mathrm{D}_{2} \mathrm{O}\right.$ exchangeable, $\left.\mathrm{NH}_{2}\right) ;{ }^{13} \mathrm{C}$ NMR (DMSO- $d_{6}, 75 \mathrm{MHz}$ ): $\delta 170.3$ (C-2), 162.5, 164.3 (C-2', $\mathrm{CONH}), 143.8,142.6,138.7,133.4,132.6,132.4,131.9$, 130.6, 128.3, 127.4, 126.8, 125.9, 124.2, 123.9, 123.6, 122.3, 121.9, 120.9, 120.6, 119.6 (Bz, C-3, C-4, C-6, C-7, C-3', C-4', C-2", C-3", C-4", C-5"), 116.8 (CN); EI-MS: $m / z 526[\mathrm{M}]^{+}(38 \%)$; Analysis Calcd for $\mathrm{C}_{26} \mathrm{H}_{14} \mathrm{~N}_{4} \mathrm{O}_{3} \mathrm{~S}_{3}$ (526.61): C, 59.30; H, 2.68; N, 10.64; S, $18.27 \%$. Found: C, 59.52; H, 2.72; N, 10.39; S, $18.44 \%$.

Ethyl 5-(2-amino-4-phenylthiophene-3-carboxamido)2-(2-oxo-2H-chromen-3-yl)thieno-[3,2- $d]$ thiazole-6carboxylate (16b)

Yellow (1,4-dioxane), yield $73 \%$ (4.18 g), mp 203-207 ${ }^{\circ} \mathrm{C}$; IR (KBr) vmax 3462-3346, 3056, 1689 $1696,1641,1632 \mathrm{~cm}^{-1}$; ${ }^{1} \mathrm{H}$ NMR (DMSO- $d_{6}, 200 \mathrm{MHz}$ ): $\delta 8.33\left(1 \mathrm{H}, \mathrm{s}, \mathrm{D}_{2} \mathrm{O}\right.$ exchangeble, $\left.\mathrm{NH}\right), 7.23-7.37(9 \mathrm{H}, \mathrm{m}$, 2Bz), 6.63 (1H, s, H-4'), 6.28 (1H, s, H-5"), $4.82(2 \mathrm{H}, \mathrm{s}$, $\mathrm{D}_{2} \mathrm{O}$ exchangeable, $\left.\mathrm{NH}_{2}\right), 4.20(2 \mathrm{H}, \mathrm{q}, J=7.39 \mathrm{~Hz}$, $\left.\mathrm{OCH}_{2} \mathrm{CH}_{3}\right), 1.14\left(3 \mathrm{H}, \mathrm{t}, J=7.39 \mathrm{~Hz}, \mathrm{OCH}_{2} \mathrm{CH}_{3}\right) ;{ }^{13} \mathrm{C}$ NMR (DMSO- $\left.d_{6}, 75 \mathrm{MHz}\right): \delta 170.2(\mathrm{C}-2), 162.5,164.0$, 
164.3 (C-2', CONH, COOEt), 143.1, 142.4, 136.1, 132.8, 131.6, 130.3, 129.6, 129.2, 128.4, 128.7, 126.7, 125.9, 125.3, 125.2, 124.9, 123.8, 123.8, 123.1, 122.9, 121.8 (Bz, C-3, C-4, C-6, C-7, C-3', C-4', C-2"', C-3", C-4", C-5"), $52.3\left(\mathrm{C}, \mathrm{OCH}_{2} \mathrm{CH}_{3}\right), 16.4\left(\mathrm{C}, \mathrm{OCH}_{2} \mathrm{CH}_{3}\right)$; EI-MS: $\mathrm{m} / z 573[\mathrm{M}]^{+}(31 \%)$; Analysis Calcd for $\mathrm{C}_{28} \mathrm{H}_{19} \mathrm{~N}_{3} \mathrm{O}_{5} \mathrm{~S}_{3}$ (573.66): C, 58.62; H, 3.34; N, 7.32; S, 16.77\%. Found: C, 58.39; H, 3.51; N, 7.48; S, 16.91\%.

\section{1. 11. General Procedure for the Synthesis of the Arylhydrazone Derivatives 18a-c}

To a solution of compound $15 \mathbf{a}(4.94 \mathrm{~g}, 0.01 \mathrm{~mol})$ in ethanol $(50 \mathrm{~mL})$ containing sodium hydroxide $(10 \mathrm{~mL}$, $10 \%$ ) any of benzenediazonium chloride, 4-chlorobenzenediazonium chloride or 4-methoxybenzenediazonium chloride [prepared through the addition of sodium nitrite solution $(0.70 \mathrm{~g}, 0.01 \mathrm{~mol})$ to a cold solution $\left(0-5{ }^{\circ} \mathrm{C}\right)$ of the appropriate aromatic amine, namely aniline $(0.93 \mathrm{~g}$, $0.01 \mathrm{~mol})$, 4-chloroaniline $(1.27 \mathrm{~g}, 0.01 \mathrm{~mol})$ or 4-methoxyaniline $(1.24 \mathrm{~g}, 0.01 \mathrm{~mol})$, dissolved in concentrated hydrochloric acid $(10 \mathrm{~mL}, 18 \mathrm{~mol})$ with continuous stirring] was added portion-wise with continuous stirring. The whole reaction mixture was stirred at room for $2 \mathrm{~h}$ and the solid product formed, in each case, was collected by filtration.

2-Cyano- $N$-[6-cyano-2-(2-oxo-2H-chromen-3-yl)thieno[3,2- $d$ ] thiazol-5-yl]-3-phenyl-4-(2-phenylhydrazono)but-2-enamide (18a)

Yellow (1,4-dioxane), yield $76 \%(4.54 \mathrm{~g}) \mathrm{mp}$ 133-137 ${ }^{\circ} \mathrm{C}$; IR (KBr) vmax 3469-3341, 3056, 2221, 2220, 1689, 1691, 1648, $1631 \mathrm{~cm}^{-1} ;{ }^{1} \mathrm{H}$ NMR (DMSO- $d_{6}$, $200 \mathrm{MHz}): \delta 10.23,8.33\left(2 \mathrm{H}, 2 \mathrm{~s}, \mathrm{D}_{2} \mathrm{O}\right.$ exchangeable, 2NH), 7.48-7.25 (14H, m, 2Bz), $5.93(1 \mathrm{H}, \mathrm{s}, \mathrm{CH}=\mathrm{N})$, $6.63\left(1 \mathrm{H}, \mathrm{s}, \mathrm{H}-4\right.$ ') $;{ }^{13} \mathrm{C}$ NMR (DMSO- $\left.d_{6}, 75 \mathrm{MHz}\right): \delta$ 173.1, $171.6(\mathrm{C}-2, \mathrm{C}=\mathrm{N}), 164.9,162.8(\mathrm{C}-2$ ', NHCO), $142.5,141.3,139.5,138.2,131.8,131.2,130.8,127.6$, $126.4,125.2,124.8,124.6,124.2,123.3,122.8,122.0$, 121.3, 120.8, 120.3, 119.8 (Bz, C-3, C-4, C-6, C-7, C-3', C-4', C-2", C-3", C-4", C-5"), 116.8, 116.4 (2CN), 90.6, $88.3(\mathrm{C}=\mathrm{C})$; EI-MS: $\mathrm{m} / z 598$ [M] $^{+}(28 \%)$; Analysis Calcd for $\mathrm{C}_{32} \mathrm{H}_{18} \mathrm{~N}_{6} \mathrm{O}_{3} \mathrm{~S}_{2}$ (598.65): C, 64.20; H, 3.03; N, 14.04; $\mathrm{S}, 10.71 \%$. Found: $\mathrm{C}, 64.49 ; \mathrm{H}, 2.97 ; \mathrm{N}, 14.22 ; \mathrm{S}$, $10.64 \%$.

4-[2-(4-Chlorophenyl)hydrazono]-2-cyano- $N$-[6-cyano-2-(2-oxo-2H-chromen-3-yl)thieno[3,2-d] thiazol-5yl]-3-phenylbut-2-enamide (18b)

Yellow crystals (1,4-dioxane), 89\% (5.63 g), mp 123-125 ${ }^{\circ} \mathrm{C}$; IR (KBr) vmax 3473-3341, 3058, 2223, 2220, 1688, 1696, 1642, $1635 \mathrm{~cm}^{-1} ;{ }^{1} \mathrm{H}$ NMR (DMSO- $d_{6}$, $200 \mathrm{MHz}): \delta 10.21,8.36\left(2 \mathrm{H}, 2 \mathrm{~s}, 2 \mathrm{NH}, \mathrm{D}_{2} \mathrm{O}\right.$ exchangeable), 7.45-7.26 (13H, m, 3Bz), $6.62(1 \mathrm{H}, \mathrm{s}, \mathrm{H}-4$ '), 5.90 $(1 \mathrm{H}, \mathrm{s}, \mathrm{CH}=\mathrm{N}) ;{ }^{13} \mathrm{C}$ NMR (DMSO- $\left.d_{6}, 75 \mathrm{MHz}\right): \delta 173.3$, $171.8(\mathrm{C}-2, \mathrm{C}=\mathrm{N}), 164.6,162.6(\mathrm{C}-2$ ', NHCO $), 142.2$,
$140.8,132.3,131.8,130.8,128.4,127.9,126.4,125.9$, $125.4,124.9,124.8,124.2,123.1,122.9,122.4,121.3$, 120.8, 120.1, 119.98 (Bz, C-3, C-4, C-6, C-7, C-3', C-4', C-2", C-3", C-4", C-5"), 116.7, 115.8 (2CN),; 88.4, 90.6 $(\mathrm{C}=\mathrm{C})$; EI-MS: $\mathrm{m} / \mathrm{z} 633$ [M] $^{+}(41 \%)$; Analysis Calcd for $\mathrm{C}_{32} \mathrm{H}_{17} \mathrm{ClN}_{6} \mathrm{O}_{3} \mathrm{~S}_{2}$ (633.10): C, 60.71; H, 2.71; N, 13.27; $\mathrm{S}$, $10.13 \%$. Found: C, 60.83 ; H, 2.83; N, 13.99; S, 10.28\%.

2-Cyano- $N$-[6-cyano-2-(2-oxo-2H-chromen-3-yl)thieno[3,2-d] thiazol-5-yl]-4-[2-(4-methoxyphenyl)hydrazono]-3-phenylbut-2-enamide (18c)

Yellow crystals (1,4-dioxane), yield 68\%, (4.27 g), mp 203-207 ${ }^{\circ} \mathrm{C}$; IR (KBr) vmax 3489-3324, 3055, 2222, 2220, 1690, 1693, 1640, $1631 \mathrm{~cm}^{-1}$; ${ }^{1} \mathrm{H}$ NMR (DMSO- $d_{6}$, $200 \mathrm{MHz}): \delta 10.25,8.33\left(2 \mathrm{H}, 2 \mathrm{~s}, \mathrm{D}_{2} \mathrm{O}\right.$ exchangeable, $2 \mathrm{NH}), 7.48-7.28(13 \mathrm{H}, \mathrm{m}, 3 \mathrm{Bz}), 6.60\left(1 \mathrm{H}, \mathrm{s}, \mathrm{H}-4^{\prime}\right), 5.88$ $(1 \mathrm{H}, \mathrm{s}, \mathrm{CH}=\mathrm{N}), 1.12\left(3 \mathrm{H}, \mathrm{s}, \mathrm{CH}_{3}\right) ;{ }^{13} \mathrm{C}$ NMR (DMSO- $d_{6}$, $75 \mathrm{MHz}): \delta$ 173.1, $171.9(\mathrm{C}-2, \mathrm{C}=\mathrm{N}), 162.8,164.9$ (C-2', NHCO), 141.6, 140.4, 132.8, 131.3, 130.9, 130.2, 129.4, $126.8,126.0,125.6,125.3,124.9,124.4,123.7,123.0$, 122.8, 121.4, 120.9, 120.3, 119.7 (Bz, C-3, C-4, C-6, C-7, C-3', C-4', C-2"', C-3", C-4", C-5'), 116.6, 116.3 $(2 \mathrm{CN}), 90.3,88.7(\mathrm{C}=\mathrm{C})$; EI-MS: $m / z 628[\mathrm{M}]^{+}(33 \%)$; Analysis Calcd for $\mathrm{C}_{33} \mathrm{H}_{20} \mathrm{~N}_{6} \mathrm{O}_{4} \mathrm{~S}_{2}(628.68): \mathrm{C}, 63.05 ; \mathrm{H}$, 3.21 ; N, 13.37; S, 10.20\%. Found: C, 63.22; H, 3.51; N, $13.53 ; \mathrm{S}, 10.42 \%$.

\section{1. 12. General Procedure for the Synthesis of the Pyridazine Derivatives 19a-c}

To a suspension of any of compound 18a $(5.98 \mathrm{~g}$, $0.01 \mathrm{~mol}), \mathbf{1 8 b}(6.33 \mathrm{~g}, 0.01 \mathrm{~mol})$ or $17 \mathbf{c}(6.28 \mathrm{~g}, 0.01$ $\mathrm{mol}$ ) in sodium ethoxide solution [prepared via dissolving metallic sodium $(0.46 \mathrm{~g}, 0.02 \mathrm{~mol})$ in absolute ethanol (60 $\mathrm{mL}$ )] was heated in a boiling water bath for $3 \mathrm{~h}$ then poured onto ice/water containing a few drops of hydrochloric acid (till $\mathrm{pH}$ 6). The solid product formed was collected by filtration.

$N$-[6-Cyano-2-(2-oxo-2H-chromen-3-yl)thieno[3,2-d] thiazol-5-yl]-3-imino-2,5-diphenyl-2,3-dihydropyridazine-4-carboxamide (19a)

Yellow crystals (1,4-dioxane), yield 82\% (4.90 g), mp 287-293 ${ }^{\circ} \mathrm{C}$; IR (KBr) vmax 3477-3321, 3053, 2220, 1689, 1691, 1648, (C=N), $1631(\mathrm{C}=\mathrm{C}) \mathrm{cm}^{-1} ;{ }^{1} \mathrm{H}$ NMR (DMSO- $\left.d_{6}, 200 \mathrm{MHz}\right): \delta 10.41,8.31\left(2 \mathrm{H}, 2 \mathrm{~s}, \mathrm{D}_{2} \mathrm{O}\right.$ exchangeable, $2 \mathrm{NH}), 7.45-7.22(14 \mathrm{H}, \mathrm{m}, 3 \mathrm{Bz}), 6.60(1 \mathrm{H}, \mathrm{s}$, H-4'), 6.02 (1H, s, H-3"); ${ }^{13} \mathrm{C}$ NMR (DMSO- $d_{6}, 75$ MHz): $\delta$ 173.0, 170.3, 169.6 (C-3, C-3", C-6"), 164.6, 162.6 (C-2', NHCO), 142.9, 140.2, 133.4, 132.6, 131.8, $131.2,130.8,128.2,127.9,126.7,125.5,124.8,124.9$, 123.0, 122.8, 122.3, 121.9, 120.9, 120.8, 119.2 (Bz, C-3, C-4, C-6, C-7, C-3', C-4', C-4", C-5"), 116.2 (CN), EI-MS: $m / z 598[\mathrm{M}]^{+}$(20\%); Analysis Calcd for $\mathrm{C}_{32} \mathrm{H}_{18} \mathrm{~N}_{6} \mathrm{O}_{3} \mathrm{~S}_{2}$ (598.65): C, 64.20; H, 3.03; N, 14.04; S, $10.71 \%$. Found: C, 64.33; H, 3.19; N, 14.38; S, 10.54\%. 
2-(4-Chlorophenyl)- $\mathrm{N}$-[6-cyano-2-(2-oxo-2H-chromen -3-yl)thieno[3,2-d] thiazol-5-yl]-3-imino-5-phenyl-2,3dihydropyridazine-4-carboxamide (19b)

Pale yellow crystals (1,4-dioxane), yield 69\% (4.12 g), mp 244-147 ${ }^{\circ} \mathrm{C}$; IR (KBr) vmax 3482-3329, 3057, 2221, 1689, 1696, 1640, $1632(\mathrm{C}=\mathrm{C}) \mathrm{cm}^{-1} ;{ }^{1} \mathrm{H}$ NMR (DMSO- $\left.d_{6}, 200 \mathrm{MHz}\right): \delta 10.20,8.38\left(2 \mathrm{H}, 2 \mathrm{~s}, \mathrm{D}_{2} \mathrm{O}\right.$ exchangeable, $2 \mathrm{NH}), 7.42-7.23(13 \mathrm{H}, \mathrm{m}, 3 \mathrm{Bz}), 7.03(1 \mathrm{H}, \mathrm{s}$, H-3"), 6.64 (1H, s, H-4'); ${ }^{13} \mathrm{C}$ NMR (DMSO- $d_{6}, 75 \mathrm{MHz}$ ): $\delta$ 173.1, 170.2, 169.7 (C-3', C-3", C-6"), 162.8, 164.3 (C-2', NHCO), 142.6, 140.2, 139.5, 138.6, 132.9, 131.8, 131.6, 130.8, 127.6, 126.4, 125.7, 125.6, 124.9, 124.8, 124.2, 123.1, 122.9, 122.4, 120.8, 120.1, 121.3, $119.9(\mathrm{Bz}$, C-3, C-4, C-6, C-7, C-3', C-4', C-4", C-5"), $116.6(\mathrm{CN})$; EI-MS: $m / z 633[\mathrm{M}]^{+}(23 \%)$; Analysis Calcd for $\mathrm{C}_{32} \mathrm{H}_{17} \mathrm{Cl}-$ $\mathrm{N}_{6} \mathrm{O}_{3} \mathrm{~S}_{2}$ (633.10): C, 60.71; H, 2.71; N, 13.27; S, 10.13\%. Found: C, 60.92; H, 2.94; N, 13.40; S, 10.45\%.

$N$-[6-Cyano-2-(2-oxo-2H-chromen-3-yl)thieno[3,2-d] thiazol-5-yl]-3-imino-2-(4-methoxyphenyl)-5-phenyl2,3-dihydropyridazine-4-carboxamide (19c)

Yellow crystals (1,4-dioxane), yield 73\% (4.58 g), mp 192-196 ${ }^{\circ} \mathrm{C}$; IR (KBr) vmax 3479-3320, 3056, 2220 , 1687, 1691, 1637, $1630 \mathrm{~cm}^{-1} ;{ }^{1} \mathrm{H}$ NMR (DMSO- $d_{6}, 200$ MHz): $\delta 10.26,8.31\left(2 \mathrm{H}, 2 \mathrm{~s}, \mathrm{D}_{2} \mathrm{O}\right.$ exchangeable, $\left.2 \mathrm{NH}\right)$, 7.23-7.45 (13H, m, 3Bz), 7.03 (1H, s, H-3"), $6.71(1 \mathrm{H}, \mathrm{s}$, $\left.\mathrm{H}-4^{\prime}\right), 3.13$ (3H, s, $\left.\mathrm{CH}_{3}\right) ;{ }^{13} \mathrm{C}$ NMR (DMSO- $d_{6}, 75 \mathrm{MHz}$ ): $\delta$ 173.5, 170.2, 169.6 (C-2', C-3", C-6"), 164.3, 163.2 (C-2', NHCO), 141.3, 140.2, 130.3, 130.2, 128.6, 128.3, 127.1, 126.9, 126.2, 125.6, 125.2, 124.6, 124.4, 123.7, 123.0, 122.8, 121.7, 120.3, 120.3, 119.2 (Bz, C-3, C-4, C-6, C-7, C-3', C-4', C-4", C-5"), 116.6 (CN), 32.6 (C, $\mathrm{OCH}_{3}$ ); EI-MS: $m / z 628[\mathrm{M}]^{+}(33 \%)$; Analysis Calcd for $\mathrm{C}_{33} \mathrm{H}_{20} \mathrm{~N}_{6} \mathrm{O}_{4} \mathrm{~S}_{2}$ (628.68): C, 63.05; H, 3.21; N, 13.37; $\mathrm{S}$, $10.20 \%$. Found: C, 63.31; H, 3.38; N, 13.42; S, 10.32\%.

\section{Conclusions}

In summary, we have shown herein that our strategy is applicable for the synthesis of a wide range of thiazole derivatives and particularly of such which are incorporated into heterocyclic cores and for fused derivatives. Compounds 10b, 13b, 18a, 19b and 19c showed the maximum anti-inflammatory activities while compounds $\mathbf{6}-\mathbf{c}, \mathbf{7 a - c}$, 8b, 10b, 13a, 16b, 19b and 19c showed high anti-ulcer activities among the synthesized compounds. The toxicity of selected compounds was studied against shrimp larvae where compounds $10 \mathbf{b}, 18 \mathbf{a}, 19 \mathrm{c}$ and $19 \mathrm{~d}$ showed to be non toxic against the tested organisms.

\section{Acknowledgement}

R. M. Mohareb would like to thank the Alexander von Humboldt Foundation in Bonn, Germany for affor- ding him regular fellowships in Germany for finance and completing his research work.

\section{References}

1. S. K. Bhatia, G. Nath, R. Tilah, S. K. Singh, Eur. J. Med. Chem. 2010, 45, 651-660.

http://dx.doi.org/10.1016/j.ejmech.2009.11.008

2. B. V. Yang, D. S. Weinstein, L. M. Doweyko, H. Gong, W. Vaccaro, T. Huynh, H. Y. Xiao, A. M. Doweyko, L. Mckay, D. A. Holloway, J. E. Somerville, S. Habte, M. Cunningham, M. McMahon, R. Townsend, D. Shuster, J. H. Dodd, S. G. Nadler, J. C. Barrish, J. Med. Chem. 2010, 53, 8241-8251. http://dx.doi.org/10.1021/jm100957a

3. F. C. Spector, L. Liang, H. Giordano, M. Sivaraja, M. G. Peterson, J. Virol. 1998, 72, 6979-6987.

http://dx.doi.org/jvi.asm.org/content/72/9/6979

4. G. C. Diego, F. Douelle, T. S. Feng, A. T. Nchinda, Y. Younis, K. L. White, Q. Wu, E. Ryan, J. N. Burrows, D. Waterson, M. J. Witty, S. Wittlin, S. A. Charman, K. Chibale, J. Med. Chem. 2011, 54, 7713-7719.

http://dx.doi.org/abs/10.1021/jm201108k

5. F. W. Bell, A. S. Cantrell, M. Hoegberg, S. R. Jaskunas, N. G. Johansson, C. L. Jordan, M. D. Kinnick, P. Lind, J. M. Morin, J. Med. Chem. 1995, 38, 4929-4936. http://dx.doi.org/10.1021/jm00025a010

6. D. E. Fink, D. S. Mortensen, S. R. Stauffer, Z. D. Aron, Chem. Biol. 1999, 6, 205-219.

http://dx.doi.org/10.1016/S1074-5521(99)80037-4

7. M. Biagetti, C. P. Leslie, A. Mazzali, C. Seri, D. A. Pizzi, J. Bentley, T. Genski, R. Di Fabio, L. Zonzini, L. Caberlotto, Bioorg. Med. Chem. Lett. 2010, 20, 4741-4744.

http://dx.doi.org/10.1016/j.bmcl.2010.06.140

8. E. W. Tilburg, P. A. M. Klein, M. D. Groote, M. W. Beukers, A. P. Jerman, Bioorg. Med. Chem. Lett. 2001, 11, 20172019.

http://dx.doi.org/10.1016/S0960-894X(01)00356-0

9. B. Umadevi, Eur. J. Med. Chem. 2007, 42, 1144-1150. http://dx.doi.org/10.1016/j.ejmech.2007.01.016

10. K. J. Wilson, C. R. Illig, N. Subasinghe, J. B. Hoffman, M. J. Rudolph, R. Soll, C. J. Molloy, R. Bone, D. Green, T. Randall, M. Zhang, F. A. Lewandowski, Z. Zhou, C. Sharp, D. Maguire, B. Grasberger, R. L. Jarlais, J. Spurlino, Bioorg. Med. Chem. Lett. 2001, 11, 915-918.

http://dx.doi.org/10.1016/S0960-894X(01)00102-0

11. W. T. Zhang, J. L. Ruan, P. F. Wu, F. C. Jiang, L. N. Zhang, W. Fang, X. L. Chen, Y. Wang, B. S. Cao, G. Y. Chen, Y. J. Zhu, J. Gu, J. G. Chen, J. Med. Chem. 2009, 52, 718-725 http://dx.doi.org/10.1021/jm800902t

12. G. Gebeyehu, V. E. Marquez, A. V. Cott, D. A. Cooney, J. A. Kelley, H. N. Jayaram, G. S. Ahluwalia, R. L. Dion, Y. A. Wilson, D. G. Johns, J. Med. Chem. 1985, 28, 99-105. http://dx.doi.org/10.1021/jm00379a018

13. A. Sekiguchi, A. Nishina, H. Kimura, R. H. Fukumoto, K. Kanoh, H. A. Ishahara, M. Koketsu, Chem. Pharm. Bull. 
2005, 53, 1439-1442.

http://dx.doi.org/10.1111/j.1742-4658.2007.06125.x

14. M. Koketsu, H. Ishihara, W. Wu, K. Murakami, I. Saiki, Eur. J. Pharm. Sci. 1999, 9, 157-161. http://dx.doi.org/10.1016/S0928-0987(99)00058-5

15. A. I. Khalaf, Al-Kadhimi, A. A. H. Ahmed, J. H. Ali, Acta Chim. Slov. 2016, 63, 689-704. http://dx.doi.org/10.17344/acsi.2016.2775

16. R. M. Mohareb, A. A. Mohamed, A. E. M. Abdallah, Acta Chim. Slov. 2016, 63, 227-240.

http://dx.doi.org/10.17344/acsi.2015.1668

17. M. I. I. Fakhr, M. A. A. Radwan, S. El-Batran, O. M. E. Abd El-Salam, S. M. El-Shenawy, Eur. J. Med. Chem. 2009, 44, 1718-1725.

http://dx.doi.org/10.1016/j.ejmech.2008.02.034

18. W. C. Shearouse, M. Z. Shumba, J. Mack, Molecules 2014, 4,171-179.

http://dx.doi.org/10.3390/app4020171
19. C. A. Winter, E. A. Risley, G. W. Nuss, Proc. Soc. Exp. Biol. Ther. 1962, 111, 544-547.

20. H. Shay, S. A. Komarov, S. S. Fels, D. Meranze, M. Grunstein, H. Siplet, Gastroenterology 1945, 5, 43-61.

21. J. P. Overmyer, D. R. Rouse, J. K. Avants, A.W. Garrison, M. E. Delorenzo, K. W. Chung, P. B. Key, W. A. Wilson, M. C. Black, J. Environ. Sci. Health 2007, 42,471-480. http://dx.doi.org/10.1080/10934520601187690

22. R. Maltais, M. A. Fournier, D. Poirier, Bioorg. Med. Chem. 2011, 19, 4652-68. http://dx.doi.org/10.1016/j.bmc.2011.06.003

23. S. Kanchithalaivan, R. R. Kumar, S. Perumal, Steroids 2013, 78, 409-417. http://dx.doi.org/10.1016/j.steroids.2012.12.017

24. G. B. Djigoué, L. C. Kenmogne, J. Roy, D. Poirier, Bioorg. Med. Chem. Lett. 2013, 23, 6360-6360. http://dx.doi.org/10.1016/j.bmcl.2013.09.072

25. M. G. Metcalf,. Clin. Biochem. 1974, 7, 119-130. http://dx.doi.org/10.1016/S0009-9120(74)91218-1

\section{Povzetek}

Reakcija 2-(4-okso-4,5-dihidrotiazol-2-il)acetonitrila (1) s salicilaldehidom (2) v 1,4-dioksanu ob dodatku katalitske množine piridina daje kumarinske derivate 3. Ob reakciji le-teh z različnimi reagenti nastanejo pirano[4,5-b]tiazolni, pirido[4,5-b]tiazolni in tieno[5,4-b]tiazolni derivati. Za novo pripravljene spojine smo raziskali učinkovitost delovanja proti vnetjem in želodčnim razjedam ter ugotovili, da so spojine 7a, 8a, 10b, 13b, 15b, 18a, 19b, 19c, in 19d pokazale bistveno večje aktivnosti kot ostale pripravljene spojine. Poleg tega smo raziskali tudi strupenost aktivnih spojin za ličinke morskih rakcev ter ugotovili, da spojine 10b, 18a, 19c in 19d ne kažejo strupenosti za testirane organizme. 